\title{
Geometric optics and instability for NLS and Davey-Stewartson models
}

Received June 30, 2010 and in revised form February 9, 2011

\begin{abstract}
We study the interaction of (slowly modulated) high frequency waves for multi-dimensional nonlinear Schrödinger equations with gauge invariant power-law nonlinearities and nonlocal perturbations. The model includes the Davey-Stewartson system in its elliptic-elliptic and hyperbolic-elliptic variants. Our analysis reveals a new localization phenomenon for nonlocal perturbations in the high frequency regime and allows us to infer strong instability results on the Cauchy problem in negative order Sobolev spaces, where we prove norm inflation with infinite loss of regularity by a constructive approach.
\end{abstract}

\section{Introduction}

\subsection{Motivation}

The Davey-Stewartson system (DS) provides a canonical description of the dynamics of weakly nonlinear two-dimensional waves interacting with a mean-field $\chi\left(t, x_{1}, x_{2}\right) \in \mathbb{R}$; see [18] for more details. In the following we shall consider

$$
\left\{\begin{array}{l}
i \partial_{t} \psi+\frac{1}{2}\left(\eta \partial_{x_{1}}^{2}+\partial_{x_{2}}^{2}\right) \psi=\left(\partial_{x_{1}} \chi+\mu|\psi|^{2}\right) \psi, \\
\left(\partial_{x_{1}}^{2}+\partial_{x_{2}}^{2}\right) \chi=\lambda \partial_{x_{1}}|\psi|^{2},
\end{array}\right.
$$

where $\left(x_{1}, x_{2}\right) \equiv x \in \mathbb{R}^{2}, t \in \mathbb{R}$, and $\lambda, \mu \in \mathbb{R}$ are some given parameters. In addition, the choice $\eta= \pm 1$ distinguishes between the so-called elliptic-elliptic and the hyperbolicelliptic variants of the DS system (see [18]). Clearly, the DS system with $\eta=+1$ and $\lambda=0$ simplifies to the cubic nonlinear Schrödinger equation (NLS), which we consider more generally in the $d$-dimensional case

$$
i \partial_{t} \psi+\frac{1}{2} \Delta \psi=\mu|\psi|^{2} \psi, \quad x \in \mathbb{R}^{d} .
$$

R. Carles: Univ. Montpellier 2 \& CNRS, UMR 5149, Mathématiques CC 051, F-34095 Montpellier; e-mail: Remi.Carles@math.cnrs.fr

E. Dumas: Univ. Grenoble 1, Institut Fourier, 100, rue des Mathématiques-BP 74, 38402 Saint Martin d'Hères Cedex, France; e-mail: Eric.Dumas@ujf-grenoble.fr

C. Sparber: Department of Mathematics, Statistics, and Computer Science, University of Illinois at Chicago, 851 South Morgan Street, Chicago, IL 60607, USA; e-mail: sparber@uic.edu

Mathematics Subject Classification (2010): Primary 35Q55, 35B40, 35A01; Secondary 35B25, $35 \mathrm{~B} 34$ 
The cubic NLS equation is a canonical model for weakly nonlinear wave propagation in dispersive media and has numerous applications in nonlinear optics, quantum superfluids, or the description of water waves (cf. [18]). We shall allow for more general, gauge invariant, nonlinearities and consider

$$
i \partial_{t} \psi+\frac{1}{2} \Delta \psi=\mu|\psi|^{2 v} \psi, \quad x \in \mathbb{R}^{d},
$$

where $v \in \mathbb{N}^{\star}=\mathbb{N} \backslash\{0\}$. For such equations, one usually distinguishes between focusing $\mu<0$ and defocusing $\mu>0$ nonlinearities. The sign of $\mu$ has a huge impact on the issue of global well-posedness, since it is well known (cf. [18] for a broad review) that for $\mu<0$ finite-time blow-up of solutions may occur for $d \geq 2$, more specifically:

$$
\lim _{t \rightarrow T^{*}}\|\nabla \psi(t, \cdot)\|_{L^{2}\left(\mathbb{R}^{d}\right)}=\infty, \quad T^{*}<+\infty
$$

Thus, in general we cannot expect global well-posedness to hold in, say, $H^{1}\left(\mathbb{R}^{d}\right)$. On the other hand, one might ask about the possibility that even local (in time) well-posedness fails. To be more precise, we recall the following definition:

Definition 1.1 (from [14]). Let $\sigma, s \in \mathbb{R}$. The Cauchy problem for (1.6) is well posed from $H^{s}\left(\mathbb{R}^{d}\right)$ to $H^{\sigma}\left(\mathbb{R}^{d}\right)$ if, for all bounded subset $B \subset H^{s}\left(\mathbb{R}^{d}\right)$, there exist $T>0$ and a Banach space $X_{T}$ continuously embedded in $C\left([0, T] ; H^{\sigma}\left(\mathbb{R}^{d}\right)\right)$ such that for all $\varphi \in H^{s}\left(\mathbb{R}^{d}\right)$, (1.6) has a unique solution $\psi \in X_{T}$ with $\psi_{\mid t=0}=\varphi$, and the mapping $\varphi \mapsto \psi$ is continuous from $\left(B,\|\cdot\|_{H^{s}}\right)$ to $C\left([0, T] ; H^{\sigma}\left(\mathbb{R}^{d}\right)\right)$.

Remark 1.2. The introduction of $X_{T}$ is due to the fact that in several known cases, uniqueness is not established in $C\left([0, T] ; H^{\sigma}\left(\mathbb{R}^{d}\right)\right)$, but in a smaller space. Typically, for dispersive equations, one often has to consider $X_{T}=C\left([0, T] ; H^{\sigma}\left(\mathbb{R}^{d}\right)\right) \cap L^{p}([0, T]$; $\left.W^{1, q}\left(\mathbb{R}^{d}\right)\right)$, for some suitable pair $(p, q)$.

The negation of the above definition is called a lack of well-posedness or instability. In order to gain a rough idea why instability occurs, we consider the Cauchy problem for (NLS) with initial data $\psi_{0} \in H^{s}\left(\mathbb{R}^{d}\right)$. Under the assumption $v \in \mathbb{N}^{\star}$, the nonlinearity is smooth, and thus local well-posedness (from $H^{s}\left(\mathbb{R}^{d}\right)$ to $H^{s}\left(\mathbb{R}^{d}\right)$ ) holds for sufficiently large $s$ ( $s>d / 2$ does the job). On the other hand, one should note that (NLS) is invariant under the Galilean transformations,

$$
\psi(t, x) \mapsto e^{i v \cdot x-i|v|^{2} t / 2} \psi(t, x-v t), \quad v \in \mathbb{R}^{d},
$$

which leave the $L^{2}\left(\mathbb{R}^{d}\right)$ norm invariant. In addition, solutions to (NLS) are invariant under the scaling transformation

$$
\psi(t, x) \mapsto \Lambda^{-1 / v} \psi\left(t / \Lambda^{2}, x / \Lambda\right), \quad \Lambda>0 .
$$

Denoting the critical exponent $s_{C}$ by

$$
s_{c}:=\frac{d}{2}-\frac{1}{v}
$$


this scaling is easily seen to leave the homogeneous Sobolev space $\dot{H}^{s_{c}}\left(\mathbb{R}^{d}\right)$ invariant and thus we heuristically expect local well-posedness to hold only in $H^{s}\left(\mathbb{R}^{d}\right)$ with $s \geq$ $\max \left\{s_{c}, 0\right\}$. The reason for this being that for $s<\max \left\{s_{c}, 0\right\}$ and sufficiently large $\Lambda>0$ we can use the scaling symmetry of (NLS) to relate the norm of large solutions at time $t>0$ to the norm of small solutions at some time $t^{*}<t$. In other words, the difference between two solutions will immediately become very large in $H^{s}\left(\mathbb{R}^{d}\right)$, even if they are close to each other initially.

For the cubic NLS equation we have $s_{c}=0$ if $d=2$, and thus instability should occur for $\psi_{0} \in H^{s}\left(\mathbb{R}^{2}\right)$ with $s<0$. Moreover, we expect the same behavior to be true also for the DS system, since (DS) can be written in the form of an NLS with nonlocal perturbation, i.e.

$$
i \partial_{t} \psi+\frac{1}{2}\left(\eta \partial_{x_{1}}^{2}+\partial_{x_{2}}^{2}\right) \psi=\lambda E\left(|\psi|^{2}\right) \psi+\mu|\psi|^{2} \psi, \quad x \in \mathbb{R}^{2} .
$$

Here the operator $E$ acting as a Fourier multiplier on $|\psi|^{2}$ is defined via

$$
\widehat{E(f)}(\xi)=\frac{\xi_{1}^{2}}{\xi_{1}^{2}+\xi_{2}^{2}} \widehat{f}(\xi), \quad\left(\xi_{1}, \xi_{2}\right)=\xi \in \mathbb{R}^{2}
$$

where $\widehat{f}$ denotes the Fourier transform of $f$, defined as

$$
(\mathcal{F} f)(\xi) \equiv \widehat{f}(\xi)=\frac{1}{(2 \pi)^{d / 2}} \int_{\mathbb{R}^{d}} f(x) e^{-i x \cdot \xi} d x .
$$

With this definition, we have $\mathcal{F}^{-1} g=\mathcal{F} \check{g}$, with $\check{g}=g(-\cdot)$. Note that the nonlocal term in (1.2) scales like the nonlinearity in the cubic NLS equation, since the kernel

$$
\widehat{K}(\xi)=\frac{\xi_{1}^{2}}{\xi_{1}^{2}+\xi_{2}^{2}} \in L^{\infty}\left(\mathbb{R}^{2}\right)
$$

is homogeneous of degree zero. We therefore expect instability of the DS system in Sobolev spaces of negative order. It will be one of the main tasks of this work to rigorously prove this type of instability, which can be seen as a negative result, complementing the well-posedness theorems of [12] (see also [11]). To this end, we shall rely on the framework of weakly nonlinear geometric optics (WNLGO), developed in [5] for NLS. We shall henceforth study, as a first step, the interaction of highly oscillatory waves within (1.2) and describe the possible (nonlinear) resonances between them. In our opinion this is interesting in itself since it generalizes the results of [5] and reveals a new localization property for nonlocal operators in the high frequency regime. Moreover, we shall see that possible resonances heavily depend on the choice of $\eta= \pm 1$.

In order to treat the DS system and the NLS equation simultaneously, we shall from now on consider the following NLS type model:

$$
i \partial_{t} \psi+\frac{1}{2} \Delta_{\eta} \psi=\lambda E\left(|\psi|^{2 v}\right) \psi+\mu|\psi|^{2 v} \psi, \quad \psi(0, x)=\psi_{0}(x),
$$


with $x \in \mathbb{R}^{d}, \lambda, \mu \in \mathbb{R}$, and $\nu \in \mathbb{N}^{\star}$ and a generalized dispersion of the form

$$
\Delta_{\eta}:=\sum_{j=1}^{d} \eta_{j} \partial_{x_{j}}^{2}, \quad \eta_{j}= \pm 1,
$$

Furthermore, we generalize the operator $E$ given in (1.3) by imposing the following assumption:

Assumption 1.3. The operator $E$ is given by

$$
E(f)=K * f, \quad K \in \mathcal{S}^{\prime}\left(\mathbb{R}^{d}\right)
$$

where $\widehat{K}$ is homogeneous of degree zero, and continuous away from the origin.

Remark 1.4. For $\lambda=0$ and nonuniform signs of the $\eta_{j}$ 's, equation (1.6) simplifies to the so-called hyperbolic NLS, which arises, for example, in the description of surface-gravity waves on deep water (cf. [18]).

\subsection{Weakly nonlinear geometric optics}

We aim to understand the interaction of high frequency waves within solutions to (1.6). To this end, we consider the following model:

$$
i \varepsilon \partial_{t} u^{\varepsilon}+\frac{\varepsilon^{2}}{2} \Delta_{\eta} u^{\varepsilon}=\varepsilon \lambda E\left(\left|u^{\varepsilon}\right|^{2 v}\right) u^{\varepsilon}+\varepsilon \mu\left|u^{\varepsilon}\right|^{2 v} u^{\varepsilon}, \quad u^{\varepsilon}(0, x)=u_{0}^{\varepsilon}(x)
$$

where $0<\varepsilon \ll 1$ denotes a small semi-classical parameter. The singular limiting regime where $\varepsilon \rightarrow 0$ yields the high frequency asymptotics for (1.6) in a weakly nonlinear scaling (note that $\varepsilon$ appears in front of the nonlinearities). The latter is known to be critical as far as geometric optics is concerned (see [4]).

As in [5], we shall assume that (1.8) is subject to initial data given by a superposition of $\varepsilon$-oscillatory plane waves, i.e.

$$
u_{0}^{\varepsilon}(x)=\sum_{j \in J_{0}} \alpha_{j}(x) e^{i \kappa_{j} \cdot x / \varepsilon}
$$

where for some index set $J_{0} \subseteq \mathbb{N}$ we are given initial wave vectors $\kappa_{j} \in \mathbb{R}^{d}$ with corresponding smooth, rapidly decaying amplitudes $\alpha_{j} \in \mathcal{S}\left(\mathbb{R}^{d} ; \mathbb{C}\right)$. Since, in general, we can allow for countably many $\alpha_{j}$ 's, we shall impose the following summability condition:

Assumption 1.5. The initial amplitudes satisfy

$$
\sum_{j \in J_{0}}\left\langle\kappa_{j}\right\rangle^{2}\left\|\widehat{\alpha}_{j}\right\|_{L^{1} \cap L^{2}}+\sum_{j \in J_{0}}\left\|\widehat{\Delta \alpha_{j}}\right\|_{L^{1} \cap L^{2}}<+\infty,
$$

where $\left\langle\kappa_{j}\right\rangle:=\left(1+\left|\kappa_{j}\right|^{2}\right)^{1 / 2}$. 
This condition will become clear in Section 4, where we justify multiphase weakly nonlinear geometric optics using the framework of Wiener algebras.

The initial condition (1.9) induces high frequency oscillations within the solution of (1.8). The first main result of this work concerns the approximation of the exact solution $u^{\varepsilon}$ of (1.8) by (possibly countably many) slowly modulated plane waves.

Theorem 1.6. Let $d \geq 1, \lambda, \mu \in \mathbb{R}$ and $\nu \in \mathbb{N}^{*}$, and let $E$ satisfy Assumption 1.3. Consider initial data of the form (1.9) with $\kappa_{j} \in \mathbb{Z}^{d}$ and $\alpha_{j} \in \mathcal{S}\left(\mathbb{R}^{d} ; \mathbb{C}\right)$ satisfying Assumption 1.5. Then there exist $T>0$, and $C, \varepsilon_{0}>0$, such that for all $\left.\left.\varepsilon \in\right] 0, \varepsilon_{0}\right]$, there exists a unique solution $u^{\varepsilon} \in C\left([0, T] ; L^{\infty} \cap L^{2}\right)$ to (1.8)-(1.9). It can be approximated by

$$
\begin{aligned}
& \sup _{t \in[0, T]}\left\|u^{\varepsilon}(t, \cdot)-u_{\mathrm{app}}^{\varepsilon}(t, \cdot)\right\|_{L^{\infty} \cap L^{2}\left(\mathbb{R}^{d}\right)} \underset{\varepsilon \rightarrow 0}{\longrightarrow} 0 \quad \text { if } \lambda \neq 0, \\
& \sup _{t \in[0, T]}\left\|u^{\varepsilon}(t, \cdot)-u_{\mathrm{app}}^{\varepsilon}(t, \cdot)\right\|_{L^{\infty} \cap L^{2}\left(\mathbb{R}^{d}\right)} \leq C \varepsilon \quad \text { if } \lambda=0 .
\end{aligned}
$$

Here, the approximate solution $u_{\mathrm{app}}^{\varepsilon} \in C\left([0, T] ; L^{\infty} \cap L^{2}\right)$ is given by

$$
u_{\mathrm{app}}^{\varepsilon}(t, x)=\sum_{j \in J} a_{j}(t, x) e^{i \phi_{j}(t, x) / \varepsilon},
$$

where the amplitudes $a_{j} \in C\left([0, T] ; L^{\infty} \cap L^{2}\left(\mathbb{R}^{d}\right)\right)$ solve the system (2.16) and the phases $\phi_{j}$ are given by

$$
\phi_{j}(t, x)=\kappa_{j} \cdot x-\frac{t}{2} \sum_{\ell=1}^{d} \eta_{\ell} \kappa_{j, \ell}^{2} .
$$

In addition the index set $J \subseteq \mathbb{N}$ can be determined from $J_{0}$ by following the approach outlined in Sections 2.1.1 and 2.2.1.

Remark 1.7. The assumption $\kappa_{j} \in \mathbb{Z}^{d}$ is introduced to avoid small divisor problems. This aspect is discussed in more detail in [5]. Following the strategy of [5], we could state a more general result here. We have chosen not to do so, for the sake of readability.

In general we have $J_{0} \subseteq J$, due to possible resonances, i.e. the creation of new (characteristic) oscillatory phases $\phi_{j}$ not originating from the given initial data but solely due to nonlinear interactions. The above theorem generalizes the results of [5], in three different directions:

1. The approximation result is extended to $L^{2} \cap L^{\infty}$ (in [5] we only proved an approximation in $L^{\infty}$ ).

2. We allow for nonelliptic Schrödinger operators corresponding to nonuniform sign for the $\eta_{j}$ 's. This yields a resonance structure which is different from the elliptic case (see Section 2.2). In particular, one should note that for $d=2, \eta_{1}=-\eta_{2}$ and $\kappa_{j}=$ $(k, k) \in \mathbb{R}^{2}$, the corresponding phase $\phi_{j}$ simplifies to $\phi_{j}(x)=k\left(x_{1}+x_{2}\right)$, describing $\varepsilon$-oscillations which do not propagate in time. 
3. In comparison to [5] we can now take into account the nonlocal Fourier multiplier $E$. This operator, roughly speaking, behaves like a local nonlinearity in the limit $\varepsilon \rightarrow 0$ (see Section 2.2.2). The behavior is therefore qualitatively different from earlier results given in [13], where it has been proven that for (slightly more regular) integral kernels $K$ such that $\langle\xi\rangle \widehat{K}(\xi) \in L^{\infty}$ no new resonant phase can be created by $E$, in contrast to our work. Notice that the present work suggests that the same conclusion also holds for the Schrödinger-Poisson system (on $\mathbb{R}^{d}, d \geq 3$, so $\widehat{K}(\xi)=c_{d} /|\xi|^{2}$ ), even though in that case, the kernel does not satisfy the above assumption; see Remark 2.10 below for more details.

Remark 1.8. Finally, we underscore that Theorem 1.6 includes other NLS type models with nonlocal perturbations than DS, provided the corresponding kernel $\widehat{K}$ is homogeneous of degree zero and continuous away from the origin. A particular example is given by the Gross-Pitaevskii equation for dipolar quantum gases, i.e.

$$
i \partial_{t} \psi+\frac{1}{2} \Delta \psi=\mu|\psi|^{2} \psi+\lambda\left(K *|\psi|^{2}\right) \psi, \quad x \in \mathbb{R}^{3},
$$

where the interaction kernel $K$ is given by

$$
K(x)=\frac{1-3 \cos ^{2} \theta}{|x|^{3}} .
$$

Here, $\theta=\theta(x)$ stands for the angle between $x \in \mathbb{R}^{3}$ and a given dipole axis $n \in \mathbb{R}^{3}$, with $|n|=1$. In other words $\theta$ is defined via $\cos \theta=n \cdot x /|x|$. In this case, we compute (see [6]), for $\xi \in \mathbb{R}^{3} \backslash\{0\}$,

$$
\widehat{K}(\xi)=\frac{2}{3}(2 \pi)^{5 / 2}\left(3 \cos ^{2} \Theta-1\right)
$$

where $\Theta$ stands for the angle between $\xi$ and the dipole axis. The model (DGP) has been introduced in [21] in order to describe (superfluid) Bose-Einstein condensates of particles with large magnetic dipole moments. Note that for our analysis, we neglect possible external potentials $V(x)$, usually present in physical experiments. In this context, rescaling (DGP) and studying the asymptotics as $\varepsilon \rightarrow 0$ correspond to the classical limit of quantum mechanics.

\subsection{Instability and norm inflation}

The insight gained in the proof of Theorem 1.6 will allow us to infer instability results of the Cauchy problem corresponding to (1.6). Let us note that the first rigorous result on the lack of well-posedness for the Cauchy problem of (NLS) in negative order Sobolev spaces can be traced back to [14], where the focusing case in $d=1$ was studied. This result was then generalized to $d \geq 1$ in [7], where the lack of well-posedness for (NLS) from $H^{s}\left(\mathbb{R}^{d}\right)$ to $H^{s}\left(\mathbb{R}^{d}\right)$, has been proved for all $s<0$ (regardless of the sign of the nonlinearity). A general approach to prove instability was given in [3], where the authors studied the quadratic NLS. Applying their abstract result [3, Proposition 1] to the models considered above, we prove a lack of well-posedness from $H^{s}\left(\mathbb{R}^{d}\right)$ to $H^{\sigma}\left(\mathbb{R}^{d}\right)$ for all $\sigma \in \mathbb{R}$. 
Proposition 1.9. For all $s<0$ and $\sigma \in \mathbb{R}$, the Cauchy problem for (NLS), with $d \geq 2$, $v \in \mathbb{N}^{\star}$ and $\mu \neq 0$ is ill-posed from $H^{s}\left(\mathbb{R}^{d}\right)$ to $H^{\sigma}\left(\mathbb{R}^{d}\right)$. The same holds true for the Cauchy problem of (DS), provided $\lambda+2 \mu \neq 0$, and for the one of (DGP), provided $(\lambda, \mu) \neq(0,0)$.

Remark 1.10. Our result excludes the case $\lambda+2 \mu=0$, which corresponds to the situation in which the DS system is known to be completely integrable (see e.g. [1]). The algebraic structure of the equation is indeed very peculiar because of the existence of a Lax pair.

The proof of Proposition 1.9 is outlined in Appendix A, following the ideas of [3]. Note, however, that this approach is not constructive as it relies on the norm inflation for the first Picard iterate. As we shall see in Section 6, weakly nonlinear geometric optics indeed allows us to infer a stronger result than the one stated above, namely norm inflation. To this end, let us recall that in [5], weakly nonlinear geometric optics was used to prove instability results for NLS equations on $\mathbb{T}^{d}$. There, we used initial data corresponding to two nonzero amplitudes $\alpha_{0}, \alpha_{1}$ (one of which carried no $\varepsilon$-oscillation). By proving a transfer of energy (inspired by the ideas from [7] and [8]) from high to low frequencies (i.e. the zero frequency in fact) we were able to deduce instability. In the present work we shall start from three nonzero initial modes, which, via nonlinear interactions, will be shown to generate the zero mode. This phenomenon is geometrically possible as soon as a multidimensional framework is considered. From this fact we shall infer norm inflation. The price to pay in this approach via WNLGO is an unnatural condition on the initial Sobolev space:

Theorem 1.11. Consider either (NLS) with $d \geq 2, \mu \neq 0$ and $v \in \mathbb{N}^{*}$, (DS) with $\lambda+2 \mu \neq 0$, or (DGP) with $(\lambda, \mu) \neq(0,0)$. We can find a sequence of initial data $\left(\varphi_{n}\right)_{n \in \mathbb{N}} \in \mathcal{S}\left(\mathbb{R}^{d}\right)$, with

$$
\left\|\varphi_{n}\right\|_{H^{-1 /(2 v)}\left(\mathbb{R}^{d}\right)} \underset{n \rightarrow \infty}{\longrightarrow} 0,
$$

and $t_{n} \rightarrow 0$ such that the solutions $\psi_{n}$ with $\psi_{n \mid t=0}=\varphi_{n}$ satisfy

$$
\left\|\psi_{n}\left(t_{n}\right)\right\|_{H^{\sigma}\left(\mathbb{R}^{d}\right)} \underset{n \rightarrow \infty}{\longrightarrow} \infty, \quad \forall \sigma \in \mathbb{R} .
$$

Unlike the approach used in [3], in the proof of Theorem 1.11, we construct explicitly the sequence $\left(\varphi_{n}\right)_{n \in \mathbb{N}}$, as well as an approximation of $\left(\psi_{n}\right)_{n \in \mathbb{N}}$ (which can be deduced from WNLGO). Note, however, that we require $d \geq 2$, since our proof demands a multidimensional setting. Also, the reason why we restrict ourselves to (NLS), (DS) and (DGP) in the above instability results is that we do not exhibit adequate initial data for (1.6) in its full generality. In [7], norm inflation for (NLS) was shown from $H^{s}$ to $H^{s}$, under the assumption $s \leq-d / 2$. Theorem 1.11 improves this previous result in three different aspects:

1. We consider a more general NLS type model, including nonlocal perturbations (in particular the DS system).

2. The range of $s$ is larger, since we assume $s \leq-1 /(2 v)$, in a setting where we always have $1 /(2 v)<d / 2$ (recall that $d \geq 2$ by assumption). 
3. The target space is larger: all the Sobolev norms become unbounded at the same time.

Remark 1.12. In the case $0<s<s_{c}$, the norm inflation result proved in [7] was improved to a loss of regularity result in $[2,19]$, after [15] in the case of the wave equation (roughly speaking, one proves norm inflation from $H^{s}$ to $H^{\sigma}$ with $\sigma>\sigma_{0}$ for some $\sigma_{0}<s$ ). In the periodic setting $x \in \mathbb{T}^{d}$, instability results (from $H^{s}\left(\mathbb{T}^{d}\right)$ to $H^{\sigma}\left(\mathbb{T}^{d}\right)$ for all $\sigma \in \mathbb{R}$ ) were proved in [8] in the case $d=1$, and then generalized to the case $d \geq 1$ in [5] (however, the phenomenon proved there is just instability, not norm inflation). Finally, we also like to mention the beautiful result by Molinet [16] in the case $x \in \mathbb{T}$ and $v=1$, and the recent result by Panthee [17] which shows that the flow map for the BBM equation fails to be continuous at the origin from $H^{s}(\mathbb{R})$ to $\mathcal{D}^{\prime}(\mathbb{R})$ for all $s<0$.

Our last result concerns the (generalized) NLS equation only, i.e. (1.6) with $\lambda=0$, where we can prove norm inflation for a larger range of Sobolev indices.

Theorem 1.13. Let $d \geq 2, v \in \mathbb{N}^{*}, \mu \in \mathbb{R}^{*}, \lambda=0$, and $s<-1 /(1+2 v)$. We can find $a$ sequence of initial data $\varphi_{n} \in \mathcal{S}\left(\mathbb{R}^{d}\right)$ with

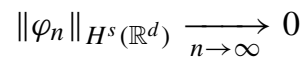

and $t_{n} \rightarrow 0$ such that the solutions $\psi_{n}$ to (1.6) with $\psi_{n \mid t=0}=\varphi_{n}$ satisfy

$$
\left\|\psi_{n}\left(t_{n}\right)\right\|_{H^{\sigma}\left(\mathbb{R}^{d}\right)} \underset{n \rightarrow \infty}{\longrightarrow}+\infty, \quad \forall \sigma \in \mathbb{R} .
$$

Remark 1.14. We believe that the restriction $s<-1 /(1+2 v)$ is only due to our approach, and we expect the result to hold under the mere assumption $s<0$. Note that for $\lambda=0$ and nonuniform signs of the $\eta_{j}$ 's in $\Delta_{\eta}$, the above result concerns the hyperbolic NLS.

To conclude this section, we point out that negative order Sobolev spaces may go against intuition. In Section 5 we shall study an asymptotic regime (for $\varepsilon \rightarrow 0$ ) where the nonlinearity is "naturally" negligible at leading order in, say, $L^{2} \cap L^{\infty}$, but fails to be negligible in some negative order Sobolev spaces. This strange behavior of negative order Sobolev spaces is further illustrated by very basic examples given in Appendix B.

Notation. Let $\left(\Lambda^{\varepsilon}\right)_{0<\varepsilon \leq 1}$ and $\left(\Upsilon^{\varepsilon}\right)_{0<\varepsilon \leq 1}$ be two families of positive real numbers.

- We write $\Lambda^{\varepsilon} \ll \Upsilon^{\varepsilon}$ if $\lim \sup _{\varepsilon \rightarrow 0} \Lambda^{\varepsilon} / \Upsilon^{\varepsilon}=0$.

- We write $\Lambda^{\varepsilon} \lesssim \Upsilon^{\varepsilon}$ if $\lim \sup _{\varepsilon \rightarrow 0} \Lambda^{\varepsilon} / \Upsilon^{\varepsilon}<\infty$.

- We write $\Lambda^{\varepsilon} \approx \Upsilon^{\varepsilon}$ (same order of magnitude) if $\Lambda^{\varepsilon} \lesssim \Upsilon^{\varepsilon}$ and $\Upsilon^{\varepsilon} \lesssim \Lambda^{\varepsilon}$.

\section{Interaction of high frequency waves in NLS type models}

In this section we shall study the interaction of high frequency waves for the generalized NLS type equation (1.6). To this end, we shall first recall some results from [5], where the usual case of NLS with elliptic dispersion is treated. 


\subsection{Geometric optics for elliptic NLS}

We consider the equation

$$
i \varepsilon \partial_{t} u^{\varepsilon}+\frac{\varepsilon^{2}}{2} \Delta u^{\varepsilon}=\varepsilon \mu\left|u^{\varepsilon}\right|^{2 v} u^{\varepsilon}, \quad x \in \mathbb{R}^{d},
$$

with $d \geq 1, \mu \in \mathbb{R}$ and $v \in \mathbb{N}^{*}$. The initial data is supposed to be given by a superposition of highly oscillatory plane waves, i.e.

$$
u^{\varepsilon}(0, x)=\sum_{j \in J_{0}} \alpha_{j}(x) e^{i \kappa_{j} \cdot x / \varepsilon},
$$

where for some index set $J_{0} \subseteq \mathbb{N}$ we are given initial wave vectors

$$
\Phi_{0}=\left\{\kappa_{j} \mid j \in J_{0}\right\}
$$

and smooth, rapidly decaying amplitudes $\alpha=\left(\alpha_{j}\right) \in \mathcal{S}\left(\mathbb{R}^{d}\right)$. We seek an approximation of the exact solution $u^{\varepsilon}$ in the following form:

$$
u^{\varepsilon}(t, x) \underset{\varepsilon \rightarrow 0}{\sim} u_{\mathrm{app}}^{\varepsilon}(t, x)=\sum_{j \in J} a_{j}(t, x) e^{i \phi_{j}(t, x) / \varepsilon} .
$$

As we shall see in the next subsection, the characteristic phases $\phi_{j}$ will be completely determined by the set of of relevant wave vectors:

$$
\Phi=\left\{\kappa_{j} \mid j \in J\right\} \supseteq \Phi_{0},
$$

In order to prove an approximation of the form (2.3), there are essentially four steps needed:

1. Derivation of the set $\Phi$.

2. Derivation of the amplitude equations, determining the $a_{j}$ 's.

3. Construction of the approximate solution.

4. Justification of the approximation.

In this section, we address the first two steps only. The last two are dealt with in Sections 3 and 4 , respectively.

2.1.1. Characteristic phases. Plugging the approximation (2.3) into (2.1), and comparing equal powers of $\varepsilon$, we find that the leading order term is of order $\mathcal{O}\left(\varepsilon^{0}\right)$. It can be made identically zero, if for all $j \in J$

$$
\partial_{t} \phi_{j}+\frac{1}{2}\left|\nabla \phi_{j}\right|^{2}=0 .
$$

This eikonal equation determines the characteristic phases $\phi_{j}(t, x) \in \mathbb{R}$, resulting in the approximation (2.3). In view of (2.2), the eikonal equation is supplemented with initial data $\phi_{j}(0, x)=\kappa_{j} \cdot x$, from which we can compute explicitly

$$
\phi_{j}(t, x)=\kappa_{j} \cdot x-\frac{t}{2}\left|\kappa_{j}\right|^{2} .
$$


Next, let $\left(\kappa_{\ell_{1}}, \ldots, \kappa_{\ell_{2 v+1}}\right)$ be a set of given wave vectors. The corresponding nonlinear interaction in $\left|u^{\varepsilon}\right|^{2 v} u^{\varepsilon}$ is then given by

$$
a_{\ell_{1}} \bar{a}_{\ell_{2}} \ldots a_{\ell_{2 v+1}} e^{i\left(\phi_{\ell_{1}}-\phi_{\ell_{2}}+\cdots+\phi_{\ell_{2 v+1}}\right) / \varepsilon}
$$

The resulting phase $\phi=\phi_{\ell_{1}}-\phi_{\ell_{2}}+\cdots+\phi_{\ell_{2 v+1}}$ satisfies the eikonal equation (2.4), and thus needs to be taken into account in our approximation, if there exists $\kappa \in \mathbb{R}^{d}$ such that

$$
\sum_{k=1}^{2 v+1}(-1)^{k+1} \kappa_{\ell_{k}}=\kappa \quad \text { and } \quad \sum_{k=1}^{2 v+1}(-1)^{k+1}\left|\kappa_{\ell_{k}}\right|^{2}=|\kappa|^{2} .
$$

With $\kappa_{j}=\kappa$, a phase $\phi_{j}$ of the form (2.5) is then said to be generated through a resonant interaction between the phases $\left(\phi_{\ell_{k}}\right)_{1 \leq k \leq 2 v+1}$.

This yields the following algorithm to construct the set $\Phi$ from $\Phi_{0}$ : Starting from the initial (at most countable) set $\Phi_{0}=\left\{\kappa_{j} \mid j \in J_{0}\right\}$, we obtain a first generation $\Phi_{1}=\left\{\kappa_{j} \mid\right.$ $\left.j \in J_{1}\right\} \supset \Phi_{0}$ (with $J_{1} \supset J_{0}$ ) by adding to $\Phi_{0}$ all points $\kappa \in \mathbb{R}^{d}$ satisfying (2.6) for some $\left\{\phi_{\ell_{1}}, \ldots, \phi_{\ell_{2 v+1}}\right\} \subset \Phi_{0}$. By a recursive scheme, we are led to a set $\Phi=\left\{\kappa_{j} \mid j \in J\right\}$ which is (at most countable and) stable under the resonance condition (2.6).

Remark 2.1. It is worth noting that $\Phi$ is a subset of the group generated by $\Phi_{0}$ (in $\left.\left(\mathbb{R}^{d},+\right)\right)$. In particular, if $\Phi_{0} \subset \mathbb{Z}^{d}$, then $\Phi \subset \mathbb{Z}^{d}$.

It turns out that we do not need a precise description of the resonances to prove norm inflation stated in Theorem 1.11 or Theorem 1.13. However, in the case of a cubic nonlinearity $v=1$, all possible resonances can be easily described geometrically by the following lemma ([10,5]). To this end, we denote for $j \in J$, the set of all resonances by

$$
I_{j}=\left\{\left.\left(\ell_{1}, \ldots, \ell_{2 v+1}\right) \in J^{2 v+1}\left|\sum_{k=1}^{2 v+1}(-1)^{k+1} \kappa_{\ell_{k}}=\kappa_{j}, \sum_{k=1}^{2 v+1}(-1)^{k+1}\right| \kappa_{\ell_{k}}\right|^{2}=\left|\kappa_{j}\right|^{2}\right\} .
$$

Lemma 2.2. Let $v=1, d \geq 2$, and $j, k, \ell, m \in J$. Then $\left(\kappa_{k}, \kappa_{\ell}, \kappa_{m}\right) \in I_{j}$ precisely when the endpoints of the vectors $\kappa_{k}, \kappa_{\ell}, \kappa_{m}, \kappa_{j}$ form four corners of a nondegenerate rectangle with $\kappa_{\ell}$ and $\kappa_{j}$ opposing each other, or when this quadruplet corresponds to one of the following two degenerate cases: $\left(\kappa_{k}=\kappa_{j}, \kappa_{m}=\kappa_{\ell}\right)$ or $\left(\kappa_{k}=\kappa_{\ell}, \kappa_{m}=\kappa_{j}\right)$.

Example 2.3. The proof of norm inflation will be based upon the following case. Let

$$
\Phi_{0}=\left\{\kappa_{1}=(1,0, \ldots, 0), \kappa_{2}=(1,1,0, \ldots, 0), \kappa_{3}=(0,1,0, \ldots, 0)\right\} \subset \mathbb{R}^{d} .
$$

The above lemma shows that for the cubic nonlinearity $(v=1)$, the set of relevant phases is simply

$$
\Phi=\Phi_{0} \cup\left\{\kappa_{0}=0_{\mathbb{R}^{d}}\right\} .
$$

One and only one phase is created by resonant interaction: the zero phase. For higher order nonlinearities $(\nu>1)$, we also have $0 \in \Phi\left(\right.$ since $\left.0=-\kappa_{1}+\kappa_{2}-\kappa_{3}+\left(\kappa_{1}-\kappa_{1}+\cdots-\kappa_{1}\right)\right)$. 
2.1.2. The amplitudes system. Continuing the formal WKB approach, the $\mathcal{O}\left(\varepsilon^{1}\right)$ term yields, after projection onto characteristic oscillations $e^{i \phi_{j} / \varepsilon}$, a system of transport equations

$$
\partial_{t} a_{j}+\kappa_{j} \cdot \nabla a_{j}=-i \mu \sum_{\left(\ell_{1}, \ldots, \ell_{2 v+1}\right) \in I_{j}} a_{\ell_{1}} \bar{a}_{\ell_{2}} \ldots a_{\ell_{2 v+1}}, \quad a_{j \mid t=0}=\alpha_{j}
$$

with the convention $\alpha_{j}=0$ if $j \notin J_{0}$. As claimed above, it is not necessary to understand the resonant sets fully to prove Theorem 1.11 or Theorem 1.13. The following lemma will suffice:

Lemma 2.4. Let $v \in \mathbb{N}^{*}, \mu \in \mathbb{R}^{*}$ and $d \geq 2$. Assume $\Phi_{0}$ is given by (2.7). There exist $\alpha_{1}, \alpha_{2}, \alpha_{3} \in \mathcal{S}\left(\mathbb{R}^{d}\right)$ such that if we set $\kappa_{0}=0_{\mathbb{R}^{d}}$, (2.8) implies

$$
\partial_{t} a_{0 \mid t=0} \neq 0
$$

For instance, this is so if $\alpha_{1}=\alpha_{2}=\alpha_{3} \neq 0$.

Proof. Assume $\alpha_{1}=\alpha_{2}=\alpha_{3}=\alpha$. Equation (2.8) yields

$$
\partial_{t} a_{0 \mid t=0}=-i \mu \sum_{\left(\ell_{1}, \ldots, \ell_{2 v+1}\right) \in I_{0}} \alpha_{\ell_{1}} \bar{\alpha}_{\ell_{2}} \ldots \alpha_{\ell_{2 v+1}}=-i \mu C(v, d)|\alpha|^{2 v} \alpha .
$$

Then, $C(v, d) \geq 1$, since $(1,2,3,1,1, \ldots, 1) \in I_{0}$.

This lemma shows that even though the zero mode is absent at time $t=0$, it appears instantaneously for a suitable choice of the initial amplitudes $\alpha_{1}, \alpha_{2}, \alpha_{3}$. This is one of the keys in the proof of the results presented in Section 1.3.

Remark 2.5. This result fails to be true in the one-dimensional cubic case $d=v=$ 1 , and in a situation where one starts with only two (nontrivial) modes $(d, v \geq 1$, but $\left.\sharp\left\{j \in J_{0} \mid \alpha_{j} \neq 0\right\} \leq 2\right)$. In both cases no new resonant mode can be created through the nonlinear interaction (see [5]).

\subsection{Geometric optics for the DS system}

In order to apply our method to the DS system (1.2), we first have to understand the resonance structure for $\eta=-1$ (a case where $\Delta_{\eta}$ is a hyperbolic operator). We shall, as a first step, neglect the action of the nonlocal term $E$ and instead consider

$$
i \varepsilon \partial_{t} u^{\varepsilon}+\frac{\varepsilon^{2}}{2}\left(\eta \partial_{x_{1}}^{2}+\partial_{x_{2}}^{2}\right) u^{\varepsilon}=\varepsilon \mu\left|u^{\varepsilon}\right|^{2} u^{\varepsilon}, \quad x \in \mathbb{R}^{2}
$$

subject to oscillatory initial data of the form (2.2). 
2.2.1. Characteristic phases and resonances. We follow the same steps as in Section 2.1.1 and determine the characteristic phases via

$$
\partial_{t} \phi+\frac{1}{2}\left(\eta\left(\partial_{x_{1}} \phi\right)^{2}+\left(\partial_{x_{2}} \phi\right)^{2}\right)=0, \quad \phi(0, x)=\kappa \cdot x .
$$

Denoting $\kappa=(p, q)$, the solution of this equation is given by

$$
\phi_{j}(t, x)=p x_{1}+q x_{2}-\frac{t}{2}\left(q^{2}+\eta p^{2}\right) .
$$

In the case $\eta=-1$ we see that if initially $\kappa=( \pm p, \pm p)$, then $\phi(t, x)=\kappa \cdot x$ is independent of time.

In order to understand possible resonances due to the cubic nonlinearity, we simply notice that, if some phases $\phi_{k}, \phi_{\ell}$ and $\phi_{m}$ are given by (2.11) (with $\kappa$ equal to $\kappa_{k}, \kappa_{\ell}$ and $\kappa_{m}$, respectively), then the combination $\phi=\phi_{k}-\phi_{\ell}+\phi_{m}$ again solves (2.10) if, and only if, it is of the form given by (2.11), with $\kappa=(p, q) \in \mathbb{R}^{2}$ satisfying

$$
\kappa=\kappa_{k}-\kappa_{\ell}+\kappa_{m}, \quad q^{2}+\eta p^{2}=q_{k}^{2}-q_{\ell}^{2}+q_{m}^{2}+\eta\left(p_{k}^{2}-p_{\ell}^{2}+p_{m}^{2}\right) .
$$

Thus, the same iterative procedure as in Section 2.1.1 allows us to build from a given (at most countable) set of wave-vectors $\Phi_{0}=\left\{\kappa_{j} \mid j \in J_{0}\right\}$ in $\mathbb{R}^{2}$, a new set $\Phi=$ $\left\{\kappa_{j} \mid j \in J\right\}$, closed under the resonance condition (2.12). Again, it is worth noting that $\Phi$ is a subset of the group generated by $\Phi_{0}$ (in $\left(\mathbb{R}^{2},+\right)$ ). In particular, if $\Phi_{0} \subset \mathbb{Z}^{2}$, then $\Phi \subset \mathbb{Z}^{2}$. We consequently alter the definition of the resonance set $I_{j}$ given above and denote, for all $j \in J$,

$I_{j}=\left\{(k, \ell, m) \in J^{3} \mid \kappa_{j}=\kappa_{k}-\kappa_{\ell}+\kappa_{m}, q_{j}^{2}+\eta p_{j}^{2}=q_{k}^{2}-q_{\ell}^{2}+q_{m}^{2}+\eta\left(p_{k}^{2}-p_{\ell}^{2}+p_{m}^{2}\right)\right\}$.

The case $\eta=1$ has already been discussed in Section 2.1.1. To understand better the nonelliptic case $\eta=-1$ we first note that (2.12) is equivalently fulfilled by $\left(\kappa_{k}-\kappa\right.$, $\left.\kappa_{\ell}-\kappa, \kappa_{m}-\kappa\right)$, as can easily be checked by a direct computation. Thus it is enough to understand the case where the zero mode $\kappa=(0,0)$ is created. In this case the resonance condition (2.12) is equivalent to

$$
\kappa_{\ell}=\kappa_{k}+\kappa_{m} \quad \text { with } \kappa_{k}, \kappa_{m} \text { satisfying } \quad q_{k} q_{m}=p_{k} p_{m} .
$$

This leads to the following statement:

Lemma 2.6. Let $\eta=-1$, and $j, k, \ell, m \in J$. Then $\left(\kappa_{k}, \kappa_{\ell}, \kappa_{m}\right) \in I_{j}$ precisely when $\left(\kappa_{k}=\kappa_{j}, \kappa_{m}=\kappa_{\ell}\right)$, or $\left(\kappa_{k}=\kappa_{\ell}, \kappa_{m}=\kappa_{j}\right)$, or when the endpoints of the vectors $\kappa_{j}$, $\kappa_{k}, \kappa_{\ell}, \kappa_{m}$ form four corners of a nondegenerate parallelogram, with $\kappa_{\ell}$ and $\kappa_{j}$ opposing each other, and such that $\left(\kappa_{k}-\kappa_{\ell}\right) /\left|\kappa_{k}-\kappa_{\ell}\right|$ and $\left(\kappa_{m}-\kappa_{\ell}\right) /\left|\kappa_{m}-\kappa_{\ell}\right|$ are symmetric with respect to the first bisector.

The resonance condition is different from the elliptic case $\eta=+1$ (Lemma 2.2, based on the completion of rectangles). In particular resonances for $\eta=+1$ are not necessarily also resonances for $\eta=-1$ and vice versa, as illustrated by the examples below. The common feature of the two cases is that it takes at least three different phases to create a new one via the cubic nonlinearity. 
Example 2.7. Let $\kappa_{k}=(2,1), \kappa_{m}=(1,2)$ and $\kappa_{\ell}=(3,3)$. Then the origin is obtained by cubic resonance in the hyperbolic case $\eta=-1$, while no new phase results of the interaction of these three phases in the elliptic case.

Example 2.8. On the other hand, if $\kappa_{k}=(0,0), \kappa_{\ell}=(1,1)$ and $\kappa_{m}=(0,2)$, we obtain $\kappa_{k}-\kappa_{\ell}+\kappa_{m}=(-1,1)$ : in the elliptic case $\eta=+1$, this is resonance, while it is not in the case $\eta=-1$.

Example 2.9. With the approach we have in mind to prove Theorem 1.11, let $\Phi_{0}$ be given by (2.7). As in the elliptic case, $\Phi=\Phi_{0} \cup\left\{0_{\mathbb{R}^{2}}\right\}$ is obtained by cubic resonance when $\eta=-1$.

2.2.2. Oscillatory structure of the nonlocal term. In order to proceed further, we need to take into account the Fourier multiplier $E$ defined in (1.3). The corresponding nonlinearity is given by

$$
F\left(u_{1}^{\varepsilon}, u_{2}^{\varepsilon}, u_{3}^{\varepsilon}\right)=E\left(u_{1}^{\varepsilon} \bar{u}_{2}^{\varepsilon}\right) u_{3}^{\varepsilon} .
$$

Having in mind (2.3) the point is to understand the rapid oscillations of

$$
F\left(a_{k} e^{i \phi_{k} / \varepsilon}, a_{\ell} e^{i \phi_{\ell} / \varepsilon}, a_{m} e^{i \phi_{m} / \varepsilon}\right),
$$

with $\phi_{k}, \phi_{\ell}$ and $\phi_{m}$ satisfying (2.11). The phase $\phi_{m}$ obviously factors out and so do the oscillations in time, since they are not affected by the action of $E$. In view of the discussion on resonances, we must understand the high frequency oscillations of

$$
E\left(a_{k} \bar{a}_{\ell} e^{i \kappa \cdot x / \varepsilon}\right), \quad \text { where } \quad \kappa=\kappa_{k}-\kappa_{\ell} .
$$

If $\kappa=0$, there is no rapid oscillation, and we can directly resume the argument of the case $E=$ Id. If $\kappa \neq 0$, we denote $b=a_{k} \bar{a}_{\ell}$ and write

$$
\begin{aligned}
e^{-i \kappa \cdot x / \varepsilon} E\left(a_{k} \bar{a}_{\ell} e^{i \kappa \cdot x / \varepsilon}\right) & =\frac{1}{(2 \pi)^{2}} \int_{\mathbb{R}^{2}} \int_{\mathbb{R}^{2}} e^{i(x-y) \cdot \xi} \widehat{K}(\xi) e^{i \kappa \cdot(y-x) / \varepsilon} b(y) d y d \xi \\
& =\frac{1}{(2 \pi \varepsilon)^{2}} \int_{\mathbb{R}^{2}} \int_{\mathbb{R}^{2}} e^{i(x-y) \cdot(\zeta-\kappa) / \varepsilon} \widehat{K}(\zeta) b(y) d y d \zeta,
\end{aligned}
$$

where we have used the fact that the function $\widehat{K}$ is 0 -homogeneous. Denote by $I^{\varepsilon}(x)$ the above integral. Applying formally the stationary phase formula yields

$$
I^{\varepsilon}(x) \underset{\varepsilon \rightarrow 0}{\sim} \widehat{K}(\kappa) b(x)=\widehat{K}\left(\kappa_{k}-\kappa_{\ell}\right) a_{k}(x) \bar{a}_{\ell}(x) .
$$

This formal argument suggests that the nonlocal operator indeed acts like a cubic nonlinearity when $\varepsilon \rightarrow 0$, and reveals a formula for the corresponding amplitude system. A rigorous proof for this argument will be given later in Section 4. For the moment, we shall proceed formally by plugging the ansatz (2.3) into

$$
i \varepsilon \partial_{t} u^{\varepsilon}+\frac{\varepsilon^{2}}{2}\left(\eta \partial_{x_{1}}^{2}+\partial_{x_{2}}^{2}\right) u^{\varepsilon}=\varepsilon \lambda E\left(\left|u^{\varepsilon}\right|^{2}\right) u^{\varepsilon}+\varepsilon \mu\left|u^{\varepsilon}\right|^{2} u^{\varepsilon}, \quad x \in \mathbb{R}^{2}
$$


The terms of order $\mathcal{O}\left(\varepsilon^{0}\right)$ are zero since all the $\phi_{j}$ 's are characteristic. For the $\mathcal{O}\left(\varepsilon^{1}\right)$ term, we project onto characteristic oscillations to obtain the following system of (nonlocal) transport equations:

$$
\begin{aligned}
\partial_{t} a_{j}+\left(\eta p_{j} \partial_{x_{1}}+q_{j} \partial_{x_{2}}\right) a_{j}= & -i \lambda \sum_{\ell \in J} E\left(\left|a_{\ell}\right|^{2}\right) a_{j} \\
& -i \lambda \sum_{\substack{(k, \ell, m) \in I_{j} \\
\ell \neq k}} \widehat{K}\left(\kappa_{k}-\kappa_{\ell}\right) a_{k} \bar{a}_{\ell} a_{m} \\
& -i \mu \sum_{(k, \ell, m) \in I_{j}} a_{k} \bar{a}_{\ell} a_{m},
\end{aligned}
$$

subject to initial data $a_{j}(0, x)=\alpha_{j}(x)$.

Remark 2.10. The above computation suggests that if $\widehat{K}$ is $M$-homogeneous with $M<0$, then the second line in (2.14) vanishes in the limit $\varepsilon>0$, since $\varepsilon^{-M}$ can be factored out. This argument is made rigorous in $\$ 4.1$. If in addition there is no local nonlinearity, i.e. $\mu=0$, then (2.14) takes the form

$$
\partial_{t} a_{j}+\kappa_{j} \cdot \nabla_{\eta} a_{j}=-i \lambda \sum_{\ell \in J} E\left(\left|a_{\ell}\right|^{2}\right) a_{j}
$$

Then the modulus of $a_{j}$ is constant along the characteristic curves, along which the above equation is of the form $\left(\partial_{t}+\kappa_{j} \cdot \nabla\right) a_{j}=a_{j} \times i \mathbb{R}$, so $\left(\partial_{t}+\kappa_{j} \cdot \nabla\right)\left|a_{j}\right|^{2}=0$. In particular, no mode is created in this case, a situation to be compared with the framework of [13], where the assumptions made on $K$ are of a different kind. An important example where this remark applies is the Schrödinger-Poisson system $(d \geq 3)$, for which $M=-2$. We therefore strongly believe that also in this case one can prove a result analogous to Theorem 1.6 (with an error rate $\mathcal{O}(\varepsilon)$, since the second line in (2.14) becomes $\mathcal{O}\left(\varepsilon^{-M}\right)=\mathcal{O}\left(\varepsilon^{2}\right)$, and we will see in Section 4.2 that nonresonant phases generate an error of order $\mathcal{O}(\varepsilon))$. However, we expect that the functional setting has to be slightly modified, since the Wiener algebra framework (used to prove Theorem 1.6) may no longer be convenient due to $\widehat{K} \notin L^{\infty}$.

Similar to Lemma 2.4, with $\Phi_{0}$ as in (2.7), we can find the corresponding initial data $\alpha_{1}, \alpha_{2}, \alpha_{3}$ in $\mathcal{S}\left(\mathbb{R}^{2}\right)$, such that the zero mode appears instantaneously, provided that $\lambda+2 \mu \neq 0$.

Lemma 2.11. Let $\eta= \pm 1$. Assume $\Phi_{0}$ is given by (2.7), and set $\kappa_{0}=0_{\mathbb{R}^{2}}$. The following are equivalent:

(i) $\lambda+2 \mu \neq 0$.

(ii) There exist $\alpha_{1}, \alpha_{2}, \alpha_{3} \in \mathcal{S}\left(\mathbb{R}^{2}\right)$ such that (2.14) implies $\partial_{t} a_{0 \mid t=0} \neq 0$.

When $\lambda+2 \mu \neq 0, \alpha_{1}, \alpha_{2}, \alpha_{3}$ are admissible if and only if there is $x \in \mathbb{R}^{2}$ such that $\alpha_{1}(x) \alpha_{2}(x) \alpha_{3}(x) \neq 0$. 
Proof. For this choice of $\Phi_{0}$, setting $\kappa_{0}=0$, we have $I_{0} \neq \emptyset$. Since the only $(k, \ell, m) \in I_{0}$ corresponding to possibly nonzero products $\alpha_{k} \bar{\alpha}_{\ell} \alpha_{m} \neq 0$ are $(1,2,3)$ and $(3,2,1)$, we have, from (2.14),

$$
\begin{aligned}
\partial_{t} a_{\left.0\right|_{t=0}} & =-i\left(\lambda\left(\widehat{K}\left(\kappa_{1}-\kappa_{2}\right)+\widehat{K}\left(\kappa_{3}-\kappa_{2}\right)\right)+2 \mu\right) \alpha_{1} \bar{\alpha}_{2} \alpha_{3} \\
& =-i\left(\lambda\left(\widehat{K}\left(\kappa_{3}\right)+\widehat{K}\left(\kappa_{1}\right)\right)+2 \mu\right) \alpha_{1} \bar{\alpha}_{2} \alpha_{3} .
\end{aligned}
$$

Now, since $p_{1} p_{3}+\eta q_{1} q_{3}=0$, one easily checks the identity $\widehat{K}\left(\kappa_{3}\right)+\widehat{K}\left(\kappa_{1}\right)=1$ and thus

$$
\partial_{t} a_{\left.0\right|_{t=0}}=-i(\lambda+2 \mu) \alpha_{1} \bar{\alpha}_{2} \alpha_{3}
$$

The lemma follows.

Remark 2.12. In the case $\lambda+2 \mu=0$ (integrable case), one can prove by induction that $\partial_{t}^{m} a_{0 \mid t=0}=0$ for all $m \in \mathbb{N}$. Thus, the zero mode does not appear, at least if we consider a smooth (analytic) setting. Note that this aspect is not attributed to our initial choice $\Phi_{0}$. If $\lambda+2 \mu=0$, the zero mode cannot appear, regardless of the precise form of $\Phi_{0}$. Indeed, grouping the sets of three phases creating the zero mode (with of course $\alpha_{0}=0$ ), we may assume that we consider only three initial phases: the point is to notice that if $\kappa_{1}-\kappa_{2}+\kappa_{3}=0$, we still have

$$
\partial_{t} a_{\left.0\right|_{t=0}}=-i\left(\lambda\left(\widehat{K}\left(\kappa_{3}\right)+\widehat{K}\left(\kappa_{1}\right)\right)+2 \mu\right) \alpha_{1} \bar{\alpha}_{2} \alpha_{3}
$$

and we conclude as in the proof of Lemma 2.11.

\subsection{Possible generalizations}

As far as geometric optics is concerned (i.e. determining the resonances and deriving the corresponding amplitude system), the above analysis can be reproduced without modification (except notations) in the case of

$$
i \varepsilon \partial_{t} u^{\varepsilon}+\frac{\varepsilon^{2}}{2} \Delta_{\eta} u^{\varepsilon}=\varepsilon \lambda\left(K *\left|u^{\varepsilon}\right|^{2 v}\right) u^{\varepsilon}+\mu \varepsilon\left|u^{\varepsilon}\right|^{2 v} u^{\varepsilon}, \quad x \in \mathbb{R}^{d}, v \in \mathbb{N},
$$

provided $\widehat{K}(\xi)$ is homogeneous of degree zero and continuous away from the origin. In this case, the characteristic phases are given by

$$
\phi_{j}(t, x)=\kappa_{j} \cdot x-\frac{t}{2} \sum_{\ell=1}^{d} \eta_{\ell} \kappa_{j, \ell}^{2}
$$

and the corresponding system of transport equations reads 


$$
\begin{aligned}
\partial_{t} a_{j}+\sum_{\ell=1}^{d} \eta_{\ell} \kappa_{j, \ell} \cdot \partial_{x_{\ell}} a_{j}= & -i \lambda \sum_{\substack{\left(\ell_{1}, \ldots, \ell_{2 v}\right) \in J J^{2 v} \\
\left(\ell_{1}, \ldots, \ell_{2 v}, j\right) \in I_{j}}} E\left(a_{\ell_{1}} \bar{a}_{\ell_{2}} \ldots \bar{a}_{\ell_{2 v}}\right) a_{j} \\
& -i \lambda \sum_{\left(\ell_{1}, \ldots, \ell_{2 v+1}\right) \in I_{j}, \ell_{2 v+1} \neq j} \widehat{K}\left(\kappa_{j}-\kappa \ell_{\ell_{2 v+1}}\right) a_{\ell_{1}} \bar{a}_{\ell_{2}} \ldots a_{\ell_{2 v+1}} \\
& -i \mu \sum_{\left(\ell_{1}, \ldots, \ell_{2 v+1}\right) \in I_{j}} a_{\ell_{1}} \bar{a}_{\ell_{2}} \ldots a_{\ell_{2 v+1}},
\end{aligned}
$$

where we denote $E(f)=K * f$ and

$$
I_{j}=\left\{\left(\ell_{1}, \ldots, \ell_{2 v+1}\right) \in J^{2 v+1} \mid \phi_{j}=\phi_{\ell_{1}}-\phi_{\ell_{2}}+\cdots+\phi_{\ell_{2 v+1}}\right\} .
$$

The only point one has to check so as to derive instability results from the geometric optics result is that there exist initial data $\alpha_{j}$ such that $\partial_{t} a_{0} \neq 0$. When $\lambda=0$, the same proof as for Lemma 2.4 yields

Lemma 2.13. Let $v \in \mathbb{N}^{*}, \lambda=0, \mu \in \mathbb{R}^{*}$ and $d \geq 2$. Assume $\Phi_{0}$ is given by (2.7). There exist $\alpha_{1}, \alpha_{2}, \alpha_{3} \in \mathcal{S}\left(\mathbb{R}^{d}\right)$ such that if we set $\kappa_{0}=0_{\mathbb{R}^{d}}$, (2.16) implies

$$
\partial_{t} a_{0 \mid t=0} \neq 0 \text {. }
$$

For instance, this is so if $\alpha_{1}=\alpha_{2}=\alpha_{3} \neq 0$.

We can also take the nonlocal term into account, at least for the cubic nonlinearity and standard "elliptic" Schrödinger operator. In the case of dimension $d=2$, for all $c \in \mathbb{R}$, introduce

$$
\mathcal{E}_{c}=\left\{L \in C\left(\mathbf{S}^{1} ; \mathbb{C}\right) \mid \forall \xi \in \mathbf{S}^{1}, L\left(\xi e^{i \pi / 2}\right)=c-L(\xi)\right\}, \quad \mathbf{S}^{1}=\{\xi|| \xi \mid=1\},
$$

with the natural identification $\mathbb{R}^{2} \simeq \mathbb{C}$. There is a one-to-one correspondence, through $L \mapsto M$, where

$$
\forall \theta \in \mathbb{R}, \quad M(\theta)=L\left(e^{i \theta}\right)-c / 2,
$$

between $\mathcal{E}_{c}$ and the space of continuous functions on $\mathbb{R}$, having the "anti" $(\pi / 2)$-periodicity symmetry $M(\theta+\pi / 2)=-M(\theta)$. Lemma 2.11 is a particular case of:

Lemma 2.14. Consider $K \in \mathcal{S}^{\prime}\left(\mathbb{R}^{d}\right)$ such that $\widehat{K} \in L^{\infty}\left(\mathbb{R}^{d}\right)$ is homogeneous of degree 0 , and continuous away from the origin, but not constant on $\mathbb{R}^{d} \backslash\{0\}$. Let $v=1$, and $\lambda, \mu \in \mathbb{R}$, with $\lambda \neq 0$. Set $\kappa_{0}=0_{\mathbb{R}^{d}}$.

1. In the case $d \geq 3$, there exist $\kappa_{1}, \kappa_{2}, \kappa_{3} \in \mathbb{R}^{d} \backslash\{0\}$ and $\alpha_{1}, \alpha_{2}, \alpha_{3} \in \mathcal{S}\left(\mathbb{R}^{d}\right)$ such that, with $\Phi_{0}=\left\{\kappa_{1}, \kappa_{2}, \kappa_{3}\right\}$, (2.16) implies $\partial_{t} a_{0 \mid t=0} \neq 0$.

2. In the case $d=2$, the following are equivalent:

(i) $\widehat{K} \notin \mathcal{E}_{-2 \mu / \lambda}$.

(ii) There exist $\kappa_{1}, \kappa_{2}, \kappa_{3} \in \mathbb{R}^{2} \backslash\{0\}$ and $\alpha_{1}, \alpha_{2}, \alpha_{3} \in \mathcal{S}\left(\mathbb{R}^{2}\right)$ such that, with $\Phi_{0}=$ $\left\{\kappa_{1}, \kappa_{2}, \kappa_{3}\right\},(2.16)$ implies $\partial_{t} a_{0 \mid t=0} \neq 0$.

Furthermore, in the cases where $\partial_{t} a_{0 \mid t=0} \neq 0$ is possible, $\alpha_{1}, \alpha_{2}, \alpha_{3}$ are admissible if and only if there is $x \in \mathbb{R}^{d}$ such that $\alpha_{1}(x) \alpha_{2}(x) \alpha_{3}(x) \neq 0$. 
Proof. Finding $\kappa_{1}, \kappa_{2}, \kappa_{3} \in \mathbb{R}^{d} \backslash\{0\}$ generating $\kappa_{0}=0$ by resonance amounts to finding two nonzero and mutually orthogonal vectors, $\kappa_{1}$ and $\kappa_{3} ; \kappa_{2}$ is then determined when forming the rectangle $\left(\kappa_{0}, \kappa_{1}, \kappa_{2}, \kappa_{3}\right)$. Once such vectors are chosen, since the only $(k, \ell, m) \in I_{0}$ corresponding to possibly nonzero products $\alpha_{k} \bar{\alpha}_{\ell} \alpha_{m}$ are $(1,2,3)$ and $(3,2,1)$, we have, from $(2.14)$,

$$
\partial_{t} a_{\left.0\right|_{t=0}}=-i\left(\lambda\left(\widehat{K}\left(\kappa_{1}-\kappa_{2}\right)+\widehat{K}\left(\kappa_{3}-\kappa_{2}\right)\right)+2 \mu\right) \alpha_{1} \bar{\alpha}_{2} \alpha_{3} .
$$

This yields $\partial_{t} a_{\left.0\right|_{t=0}} \neq 0$ if and only if we are able to find two nonzero and mutually orthogonal vectors $\kappa\left(=\kappa_{1}-\kappa_{2}\right)$ and $\kappa^{\prime}\left(=\kappa_{3}-\kappa_{2}\right)$ such that $\widehat{K}(\kappa)+\widehat{K}\left(\kappa^{\prime}\right) \neq-2 \mu / \lambda$ (and in this case, the choice for $\alpha_{1}, \alpha_{2}, \alpha_{3}$ is clear).

In dimension $d=2$, the possibility of finding such $\kappa, \kappa^{\prime}$ is equivalent to requiring $K \notin \mathcal{E}_{-2 \mu / \lambda}$.

In dimension $d \geq 3$, suppose that for all choice of nonzero and mutually orthogonal $\kappa$ and $\kappa^{\prime}$, the restriction of $\widehat{K}$ to the circle $\mathbf{S}^{1}$ centered at the origin, in the plane defined by $\left\{\kappa, \kappa^{\prime}\right\}$, belongs to $\mathcal{E}_{c}$, with $c=-2 \mu / \lambda$. Choosing a direction orthogonal to both $\kappa$ and $\kappa^{\prime}$ defines the "north pole" of an $\mathbf{S}^{2}$ sphere with the above circle as equator. Then, the value of $\widehat{K}$ at this pole must be $c-\widehat{K}(\kappa)$, as well as $c-\widehat{K}\left(\kappa^{\prime}\right)$, which implies $\widehat{K}\left(\kappa^{\prime}\right)=\widehat{K}(\kappa)$, and since $\widehat{K}\left(\kappa^{\prime}\right)=c-\widehat{K}(\kappa)$, we find that $\widehat{K}$ is constant (equal to $c / 2$ ), and thus a contradiction.

This setting entails the case of the Gross-Pitaevskii equation for dipolar quantum gases (DGP), for which $d=3$. One can also easily generalize this result to higher-order nonlinearities $(v>1)$, at least in dimension $d \geq 3$. Combining nonelliptic Schrödinger operators with higher-order nonlinearities and nonlocal perturbations would lead to more tedious computations and we henceforth do not go into any further detail.

\section{Construction of the exact and approximate solutions}

\subsection{Analytical framework}

We first need to introduce the Wiener algebra framework similarly to what is used in [5].

Definition 3.1 (Wiener algebra). We define

$$
W\left(\mathbb{R}^{d}\right)=\left\{f \in \mathcal{S}^{\prime}\left(\mathbb{R}^{d} ; \mathbb{C}\right) \mid\|f\|_{W}:=\|\widehat{f}\|_{L^{1}\left(\mathbb{R}^{d}\right)}<+\infty\right\} .
$$

The space $W$ enjoys the following elementary properties (see $[9,5])$ :

1. $W$ is a Banach space, continuously embedded into $L^{\infty}\left(\mathbb{R}^{d}\right)$.

2. $W$ is an algebra, and the mapping $(f, g) \mapsto f g$ is continuous from $W^{2}$ to $W$, with

$$
\|f g\|_{W} \leq\|f\|_{W}\|g\|_{W}, \quad \forall f, g \in W .
$$

3. For all $t \in \mathbb{R}$, the free Schrödinger group

$$
U^{\varepsilon}(t)=\exp \left(i \varepsilon \frac{t}{2} \Delta_{\eta}\right)
$$

is unitary on $W$. 
In the following we shall seek an approximation result in $W \cap L^{2}=\mathcal{F}\left(L^{1}\right) \cap \mathcal{F}\left(L^{2}\right)$. The basic idea is to prove the result in $W$ only and then infer the corresponding statement in $L^{2}$. We shall therefore use the extra properties:

(2a) By the Plancherel formula and Young's inequality (or simply $\|f\|_{L^{\infty}} \leq\|f\|_{W}$ )

$$
\forall f \in W, \forall g \in L^{2}\left(\mathbb{R}^{d}\right), \quad\|f g\|_{L^{2}\left(\mathbb{R}^{d}\right)} \leq\|f\|_{W}\|g\|_{L^{2}\left(\mathbb{R}^{d}\right)} .
$$

(3a) For all $t \in \mathbb{R}$, the free Schrödinger group $U^{\varepsilon}(t)$ is unitary on $L^{2}\left(\mathbb{R}^{d}\right)$.

\subsection{Existence results}

We first treat the case of the exact solution $u^{\varepsilon}$, and then address the construction of the approximate solution $u_{\text {app }}^{\varepsilon}$.

Lemma 3.2. Consider for $x \in \mathbb{R}^{d}$ the initial value problem

$$
i \varepsilon \partial_{t} u^{\varepsilon}+\frac{\varepsilon^{2}}{2} \Delta_{\eta} u^{\varepsilon}=\varepsilon \lambda\left(K *\left|u^{\varepsilon}\right|^{2 v}\right) u^{\varepsilon}+\mu \varepsilon\left|u^{\varepsilon}\right|^{2 v} u^{\varepsilon}, \quad u^{\varepsilon}(0, x)=u_{0}^{\varepsilon}(x),
$$

where $v \in \mathbb{N}, \lambda, \mu \in \mathbb{R}$, and $K \in \mathcal{S}^{\prime}\left(\mathbb{R}^{d}\right)$ is such that $\widehat{K} \in L^{\infty}\left(\mathbb{R}^{d}\right)$. If the initial data satisfies $u_{0}^{\varepsilon} \in W \cap L^{2}$, then there exist $T^{\varepsilon}>0$ and a unique solution $u^{\varepsilon} \in C\left(\left[0, T^{\varepsilon}\right] ; W \cap L^{2}\right)$ to $(3.1)$.

Proof. The existence of a unique local in time solution in $W$ follows by combining the results of [5, Proposition 5.8] and [13, Lemma 3.3]. In both cases property (2) of Definition 3.1 together with the fact that $\widehat{K} \in L^{\infty}\left(\mathbb{R}^{d}\right)$ implies that the nonlinearities are locally Lipschitz and the result then follows by a standard fixed point argument. The existence of an $L^{2}$ solution then follows from the fact that for all $t \in\left[0, T^{\varepsilon}\right], u^{\varepsilon}(t, \cdot) \in L^{\infty}\left(\mathbb{R}^{d}\right)$, since $W \hookrightarrow L^{\infty}$. Therefore $\left|u^{\varepsilon}\right|^{2 v} \in L^{\infty}$ can be viewed as a bounded perturbation potential, and using Plancherel formula we have

$$
\begin{aligned}
\left\|\left(K *|u|^{2 v}\right) v\right\|_{L^{2}} & \leq\left\|K *|u|^{2 v}\right\|_{L^{\infty}}\|v\|_{L^{2}} \leq\left\|K *|u|^{2 v}\right\|_{W}\|v\|_{L^{2}} \\
& \leq\|\widehat{K}\|_{L^{\infty}}\left\|\widehat{|u|^{2 v}}\right\|_{L^{1}}\|v\|_{L^{2}} \leq\|\widehat{K}\|_{L^{\infty}}\|u\|_{W}^{2 v}\|v\|_{L^{2}} .
\end{aligned}
$$

Thus also the nonlocal term can be seen like a bounded perturbation and the existence then follows by standard semigroup theory.

We now pass to an existence result for the transport system (2.14). To this end we define the following space for the amplitudes.

Definition 3.3. Define

$$
X\left(\mathbb{R}^{d}\right)=\left\{a=\left(a_{j}\right)_{j \in J} \mid\left(\widehat{a}_{j}\right)_{j \in J} \in \ell^{1}\left(J ; L^{1} \cap L^{2}\left(\mathbb{R}^{d}\right)\right)\right\},
$$

equipped with the norm

$$
\|a\|_{X\left(\mathbb{R}^{d}\right)}=\sum_{j \in J}\left(\left\|\widehat{a}_{j}\right\|_{L^{1}}+\left\|\widehat{a}_{j}\right\|_{L^{2}}\right) .
$$


For $s \in \mathbb{N}$, we define

$$
X^{s}\left(\mathbb{R}^{d}\right)=\left\{a \in X\left(\mathbb{R}^{d}\right) \mid\left(\left\langle\kappa_{j}\right\rangle^{s} a_{j}\right)_{j \in J} \in X\left(\mathbb{R}^{d}\right) \text { and } \partial_{x}^{\beta} a \in X\left(\mathbb{R}^{d}\right), \forall|\beta| \leq s\right\},
$$

equipped with the norm

$$
\|a\|_{X^{s}\left(\mathbb{R}^{d}\right)}=\left\|\left(\left\langle\kappa_{j}\right\rangle^{s} a_{j}\right)_{j \in J}\right\|_{X\left(\mathbb{R}^{d}\right)}+\sum_{|\beta| \leq s}\left\|\partial_{x}^{\beta} a\right\|_{X\left(\mathbb{R}^{d}\right)} .
$$

We can state the following local in time existence result.

Lemma 3.4. Let $d \geq 1, v \in \mathbb{N} \backslash\{0\}, \lambda, \mu \in \mathbb{R}$, and $K \in \mathcal{S}^{\prime}\left(\mathbb{R}^{d}\right)$ such that $\widehat{K} \in L^{\infty}\left(\mathbb{R}^{d}\right)$. For all $\alpha=\left(\alpha_{j}\right)_{j \in J} \in X\left(\mathbb{R}^{d}\right)$, there exist $T>0$ and a unique solution

$$
t \mapsto a(t)=\left(a_{j}(t)\right)_{j \in J} \in C\left([0, T] ; X\left(\mathbb{R}^{d}\right)\right)
$$

to the transport system (2.16) with $a(0, x)=\alpha(x)$. Furthermore, the total mass is conserved:

$$
\frac{d}{d t} \sum_{j \in J}\left\|a_{j}(t)\right\|_{L^{2}}^{2}=0 .
$$

Proof. This result follows from the arguments given in [5] (see [5, Lemma 3.1] for the last point). The main aspect to remark is that the action of the nonlinear term $E$ raises no new difficulty, in view of the estimate

$$
\begin{aligned}
\left\|E\left(a_{1} \bar{a}_{2} \ldots \bar{a}_{2 v}\right) a_{2 v+1}\right\|_{X}= & \left\|\mathcal{F}\left(E\left(a_{1} \bar{a}_{2} \ldots \bar{a}_{2 v}\right)\right) * \widehat{a}_{2 v+1}\right\|_{L^{1}} \\
& +\left\|\mathcal{F}\left(E\left(a_{1} \bar{a}_{2} \ldots \bar{a}_{2 v}\right)\right) * \widehat{a}_{2 v+1}\right\|_{L^{2}} \\
\leq & \left\|\mathcal{F}\left(E\left(a_{1} \bar{a}_{2} \ldots \bar{a}_{2 v}\right)\right)\right\|_{L^{1}}\left\|a_{2 v+1}\right\|_{X}
\end{aligned}
$$

by Young's inequality. Since $\widehat{E(f g)}=(2 \pi)^{-d / 2} \widehat{K}(\widehat{f} * \widehat{g})$, we obtain

$$
\left\|E\left(a_{1} \bar{a}_{2} \ldots \bar{a}_{2 v}\right) a_{2 v+1}\right\|_{X} \leq\|\widehat{K}\|_{L^{\infty}}\left\|a_{1}\right\|_{X} \ldots\left\|a_{2 v+1}\right\|_{X} .
$$

Since the same result holds true for $E=\mathrm{Id}$ this shows that the nonlinearity on the right hand side of (2.14) defines a continuous mapping from $X\left(\mathbb{R}^{d}\right)^{2 v+1}$ to $X\left(\mathbb{R}^{d}\right)$. The existence of a local in-time solution then follows by a standard Cauchy-Lipschitz argument in the same way as in [5].

At this stage, we have constructed the approximate solution

$$
u_{\mathrm{app}}^{\varepsilon}(t, x)=\sum_{j \in J} a_{j}(t, x) e^{i \phi_{j}(t, x) / \varepsilon},
$$

where the set $J$ and the corresponding $\phi_{j}$ 's are as constructed in Section 2.1.1 and Section 2.2.1, respectively, and the profiles are given by Lemma 3.4. Since

$$
\left(a_{j}\right)_{j \in J} \in C\left([0, T] ; X\left(\mathbb{R}^{d}\right)\right),
$$

we have in particular

$$
u_{\text {app }}^{\varepsilon} \in C\left([0, T] ; W \cap L^{2}\left(\mathbb{R}^{d}\right)\right) .
$$

Higher regularity will be needed in the justification of geometric optics. 
Lemma 3.5. Under the same assumption as in Lemma 3.4 we have:

1. If $\alpha \in X^{s}\left(\mathbb{R}^{d}\right)$ for $s \in \mathbb{N}$, then the conclusions of Lemma 3.4 remain true with $X\left(\mathbb{R}^{d}\right)$ replaced by $X^{s}\left(\mathbb{R}^{d}\right)$.

2. Let $\alpha \in X^{2}\left(\mathbb{R}^{d}\right)$. Then in addition

$$
t \mapsto a(t)=\left(a_{j}(t)\right)_{j \in J} \in C^{1}\left([0, T] ; X\left(\mathbb{R}^{d}\right)\right) .
$$

Proof. The first point is straightforward. The second point stems from the first one, in view of the transport equations, (2.8), (2.14), respectively.

Remark 3.6. In particular, if the initial profiles $\left(\alpha_{j}\right)_{j \in J_{0}}$ belong to the Schwartz class, then $\left(a_{j}\right)_{j \in J} \in C\left([0, T] ; X^{s}\left(\mathbb{R}^{d}\right)\right)$ for all $s \in \mathbb{N}$.

\section{Justification of multiphase geometric optics}

In this section we justify the multiphase geometric optics approximation. We assume

$$
u^{\varepsilon}(0, x)=u_{\mathrm{app}}^{\varepsilon}(0, x)=\sum_{j \in J_{0}} \alpha_{j}(x) e^{i \kappa_{j} \cdot x / \varepsilon},
$$

with $\left(\alpha_{j}\right)_{j \in J_{0}} \in X\left(\mathbb{R}^{d}\right)$. Section 3 provides an approximate solution $u_{\mathrm{app}}^{\varepsilon} \in C([0, T], W \cap$ $L^{2}\left(\mathbb{R}^{d}\right)$ ). With this existence time $T$ (independent of $\varepsilon$ ), we prove:

Theorem 4.1. Let $d \geq 1, v \in \mathbb{N}, \lambda, \mu \in \mathbb{R}$, and E satisfy Assumption 1.3. Let $\Phi_{0} \subset \mathbb{Z}^{d}$, with corresponding amplitudes $\left(\alpha_{j}\right)_{j \in J_{0}} \in X^{2}\left(\mathbb{R}^{d}\right)$. Then there exists $\varepsilon_{0}>0$ such that for any $0<\varepsilon \leq \varepsilon_{0}$, the solution to the Cauchy problem (1.8)-(1.9) satisfies $u^{\varepsilon} \in$ $L^{\infty}\left([0, T] ; W \cap \bar{L}^{2}\right)$. In addition, $u_{\mathrm{app}}^{\varepsilon}$ approximates $u^{\varepsilon}$ in the sense that

$$
\left\|u^{\varepsilon}-u_{\mathrm{app}}^{\varepsilon}\right\|_{L^{\infty}\left([0, T] ; L^{\infty} \cap L^{2}\right)} \leq\left\|u^{\varepsilon}-u_{\mathrm{app}}^{\varepsilon}\right\|_{L^{\infty}\left([0, T] ; W \cap L^{2}\right)} \underset{\varepsilon \rightarrow 0}{\longrightarrow} 0 .
$$

When $\lambda=0, u_{\mathrm{app}}^{\varepsilon}$ approximates $u^{\varepsilon}$ up to $\mathcal{O}(\varepsilon)$ :

$$
\left\|u^{\varepsilon}-u_{\mathrm{app}}^{\varepsilon}\right\|_{L^{\infty}\left([0, T] ; L^{\infty} \cap L^{2}\right)} \leq\left\|u^{\varepsilon}-u_{\mathrm{app}}^{\varepsilon}\right\|_{L^{\infty}\left([0, T] ; W \cap L^{2}\right)} \lesssim \varepsilon .
$$

In the case $\lambda=0$, a rate $\mathcal{O}(\varepsilon)$ is available in the error estimate, while no rate should be expected in the case $\lambda \neq 0$. In the equation satisfied by $u^{\varepsilon}-u_{\text {app }}^{\varepsilon}$, the source term denoted by $r_{1}^{\varepsilon}$ below is proven to be $o(1)$ as $\varepsilon \rightarrow 0$, and no rate must be expected in general, as pointed out in Remark 4.4 below.

Remark 4.2. The above result can be proven (in the same way) in cases where the initial set of phases is not necessarily supported in $\mathbb{Z}^{d}$. We choose to prove the approximation result in this peculiar framework since it is sufficient to infer Theorems 1.11 and 1.13. A more general case would lead to small divisors problems, which can be treated as in [5]. 
Let $w^{\varepsilon}=u^{\varepsilon}-u_{\text {app }}^{\varepsilon}$ be the error term. From Section 3.2, we know that there exists $T^{\varepsilon}>0$ such that

$$
w^{\varepsilon} \in C\left(\left[0, T^{\varepsilon}\right] ; W \cap L^{2}\right) .
$$

We have to prove that for $\varepsilon$ sufficiently small, $w^{\varepsilon} \in C\left([0, T] ; W \cap L^{2}\right)$, where $T>0$ stems from Lemma 3.4, together with (4.1). A standard continuity argument shows that it suffices to prove (4.1). We compute

$$
i \varepsilon \partial_{t} w^{\varepsilon}+\frac{\varepsilon^{2}}{2} \Delta_{\eta} w^{\varepsilon}=\varepsilon\left(G\left(u^{\varepsilon}, \ldots, u^{\varepsilon}\right)-G\left(u_{\mathrm{app}}^{\varepsilon}, \ldots, u_{\mathrm{app}}^{\varepsilon}\right)\right)+\lambda \varepsilon r_{1}^{\varepsilon}+\varepsilon r_{2}^{\varepsilon}+\varepsilon r_{3}^{\varepsilon},
$$

where

$$
G\left(u_{1}, \ldots, u_{2 v+1}\right)=\lambda\left(K *\left(u_{1} \bar{u}_{2} \ldots \bar{u}_{2 v}\right)\right) u_{2 v+1}+\mu u_{1} \bar{u}_{2} \ldots u_{2 v+1},
$$

and the remainder terms are given by

$$
\begin{aligned}
r_{1}^{\varepsilon} & =\sum_{\substack{\left(\ell_{1}, \ldots, \ell_{2 v+1}\right) \in I_{j} \\
\ell_{2 v+1} \neq j}}\left(K *\left(a_{\ell_{1}} \bar{a}_{\ell_{2}} \ldots \bar{a}_{\ell_{2 v}} e^{i\left(\phi_{\ell_{1}}-\phi_{\ell_{2}}+\cdots-\phi_{\ell_{2 v}}\right) / \varepsilon}\right) a_{\ell_{2 v+1}} e^{i \phi_{\ell_{2 v+1}} / \varepsilon}\right. \\
& \left.-\widehat{K}\left(\kappa_{j}-\kappa_{\ell_{2 v+1}}\right) a_{\ell_{1}} \bar{a}_{\ell_{2}} \ldots a_{\ell_{2 v+1}} e^{i \phi_{j} / \varepsilon}\right), \\
r_{2}^{\varepsilon} & =G\left(u_{\mathrm{app}}^{\varepsilon}, \ldots, u_{\mathrm{app}}^{\varepsilon}\right)-\sum_{j \in J} \sum_{\left(\ell_{1}, \ldots, \ell_{2 v+1}\right) \in I_{j}} G\left(a_{\ell_{1}} e^{i \phi_{\ell_{1}} / \varepsilon}, \ldots, a_{\ell_{2 v+1}} e^{i \phi_{\ell_{2 v+1}} / \varepsilon}\right), \\
r_{3}^{\varepsilon} & =-\frac{\varepsilon}{2} \sum_{j \in J} e^{i \phi_{j} / \varepsilon} \Delta_{\eta} a_{j} .
\end{aligned}
$$

The term $r_{1}^{\varepsilon}$ corresponds to the stationary phase argument performed formally in Section 2.2.2, and is proven to be $o(1)$ in Lemma 4.3. The term $r_{2}^{\varepsilon}$ is more standard, and corresponds to the error introduced by non-resonant phases. Its contribution is proven to be $\mathcal{O}(\varepsilon)$ in Section 4.2 , by a suitable integration by parts. Finally, the term $r_{3}^{\varepsilon}$ corresponds to the fact that the approximate solution was constructed by canceling the $\mathcal{O}(1)$ and $\mathcal{O}(\varepsilon)$ terms only in the formal WKB construction: $r_{3}^{\varepsilon}$ corresponds to the remaining $\mathcal{O}\left(\varepsilon^{2}\right)$ terms, and is (rather obviously) of order $\mathcal{O}(\varepsilon)$.

\subsection{Localizing the nonlocal oscillations}

Lemma 4.3. Under the assumptions of Theorem 4.1, we have

$$
\left\|r_{1}^{\varepsilon}\right\|_{L^{\infty}\left([0, T] ; W \cap L^{2}\right)}^{\longrightarrow} \underset{\varepsilon \rightarrow 0}{\longrightarrow} 0 .
$$

Proof. Let $j \in J,\left(\ell_{1}, \ldots, \ell_{2 v+1}\right) \in I_{j}$, with $\kappa_{\ell_{2 v+1}} \neq \kappa_{j}$. Denote

$$
\begin{aligned}
A & =a_{\ell_{1}} \bar{a}_{\ell_{2}} \ldots \bar{a}_{\ell_{2 v}}, \quad a=a_{\ell_{2 v+1}}, \quad \kappa=\kappa_{\ell_{2 v+1}}, \\
b^{\varepsilon}(x) & =E\left(A(x) e^{i\left(\kappa_{j}-\kappa\right) \cdot x / \varepsilon}\right) a(x) e^{i \kappa \cdot x / \varepsilon}, \\
b_{\mathrm{app}}^{\varepsilon}(x) & =\widehat{K}\left(\kappa_{j}-\kappa\right) A(x) a(x) e^{i \kappa_{j} \cdot x / \varepsilon},
\end{aligned}
$$


where we have dropped the dependence upon $j, \ell_{1}, \ldots, \ell_{2 v+1}$ and $t$. Then

$$
r_{1}^{\varepsilon}=\sum_{j \in J} \sum_{\left(\ell_{1}, \ldots, \ell_{2 v+1}\right) \in I_{j}, \ell_{2 v+1} \neq j}\left(b^{\varepsilon}-b_{\text {app }}^{\varepsilon}\right) e^{i t \partial_{t} \phi_{j} / \varepsilon},
$$

where the notation $t \partial_{t} \phi_{j}$ is there only to avoid a discussion on the $\eta$ 's. We need to estimate $r_{1}^{\varepsilon}$ in $W=\mathcal{F}\left(L^{1}\right)$ and $L^{2}=\mathcal{F}\left(L^{2}\right)$, so we compute:

$$
\begin{aligned}
\widehat{b}^{\varepsilon}(\xi) & =\mathcal{F}\left(E\left(A e^{i\left(\kappa_{j}-\kappa\right) \cdot x / \varepsilon}\right) a e^{i \kappa \cdot x / \varepsilon}\right)(\xi) \\
& =(2 \pi)^{-d / 2}\left(\mathcal{F}\left(E\left(A e^{i\left(\kappa_{j}-\kappa\right) \cdot x / \varepsilon}\right)\right) * \mathcal{F}\left(a e^{i \kappa \cdot x / \varepsilon}\right)\right)(\xi) \\
& =(2 \pi)^{-d / 2} \int \widehat{K}(\zeta) \widehat{A}\left(\zeta-\frac{\kappa_{j}-\kappa}{\varepsilon}\right) \widehat{a}\left(\xi-\zeta-\frac{\kappa}{\varepsilon}\right) d \zeta \\
& =(2 \pi)^{-d / 2} \int \widehat{K}\left(\zeta+\frac{\kappa_{j}-\kappa}{\varepsilon}\right) \widehat{A}(\zeta) \widehat{a}\left(\xi-\zeta-\frac{\kappa_{j}}{\varepsilon}\right) d \zeta .
\end{aligned}
$$

On the other hand,

$$
\widehat{b}_{\text {app }}^{\varepsilon}(\xi)=(2 \pi)^{-d / 2} \int \widehat{K}\left(\kappa_{j}-\kappa\right) \widehat{A}(\zeta) \widehat{a}\left(\xi-\zeta-\frac{\kappa_{j}}{\varepsilon}\right) d \zeta .
$$

Since $\widehat{K}$ is homogeneous of degree zero, we infer

$$
\widehat{b}^{\varepsilon}(\xi)-\widehat{b}_{\text {app }}^{\varepsilon}(\xi)=(2 \pi)^{-d / 2} \int\left(\widehat{K}\left(\kappa_{j}-\kappa+\varepsilon \zeta\right)-\widehat{K}\left(\kappa_{j}-\kappa\right)\right) \widehat{A}(\zeta) \widehat{a}\left(\xi-\zeta-\frac{\kappa_{j}}{\varepsilon}\right) d \zeta .
$$

Therefore,

$$
\begin{aligned}
\left\|r_{1}^{\varepsilon}\right\|_{W \cap L^{2}} \leq \sum_{j \in J} \sum_{\left(\ell_{1}, \ldots, \ell_{2 v+1}\right) \in I_{j}, \ell_{2 v+1} \neq j} \int_{\mathbb{R}^{d}}\left|\widehat{K}\left(\kappa_{j}-\kappa_{\ell_{2 v+1}}+\varepsilon \zeta\right)-\widehat{K}\left(\kappa_{j}-\kappa_{\ell_{2 v+1}}\right)\right| \\
\cdot\left|\mathcal{F}\left(a_{\ell_{1}} \bar{a}_{\ell_{2}} \ldots \bar{a}_{\ell_{2 v}}\right)(\zeta)\right|\left\|a_{\ell_{2 v+1}}\right\|_{W \cap L^{2}} d \zeta .
\end{aligned}
$$

To conclude, we note that $\widehat{K} \in L^{\infty}\left(\mathbb{R}^{d}\right)$, and $\widehat{K}$ is continuous at $\kappa_{j}-\kappa_{\ell_{2 v+1}} \neq 0$. We can then conclude by the Dominated Convergence Theorem.

Remark 4.4. The proof shows that, in general, we cannot expect a rate in our asymptotic error estimate. For instance, for the nonlocal interaction in (DS), if $\kappa=(p, 0), p \neq 0$,

$$
\widehat{K}(\kappa+\zeta \varepsilon)-\widehat{K}(\kappa)=\frac{-\varepsilon^{2} \zeta_{2}^{2}}{\left(p+\varepsilon \zeta_{1}\right)^{2}+\varepsilon^{2} \zeta_{2}^{2}}
$$

There is no uniform control (in $\zeta$ ) other than

$$
|\widehat{K}(\kappa+\zeta \varepsilon)-\widehat{K}(\kappa)| \leq 1 .
$$




\subsection{Filtering the noncharacteristic oscillations}

Nonlinear interactions not only produce resonances, but also other noncharacteristic high frequency oscillations. The latter have to be filtered via an integration by parts. This becomes clear on the integral formulation for

$$
i \varepsilon \partial_{t} w^{\varepsilon}+\frac{\varepsilon^{2}}{2} \Delta_{\eta} w^{\varepsilon}=F^{\varepsilon},
$$

which reads

$$
w^{\varepsilon}(t, x)=U^{\varepsilon}(t) w^{\varepsilon}(0, x)-i \varepsilon^{-1} \int_{0}^{t} U^{\varepsilon}(t-\tau) F^{\varepsilon}(\tau, x) d \tau .
$$

The main result of this subsection is:

Proposition 4.5. Let $\left(\alpha_{j}\right)_{j \in J} \in X^{2}\left(\mathbb{R}^{d}\right)$. Denote

$$
R_{2}^{\varepsilon}(t, x)=-i \int_{0}^{t} U^{\varepsilon}(t-\tau) r_{2}^{\varepsilon}(\tau, x) d \tau .
$$

There exists $C>0$ such that for all $\varepsilon \in] 0,1]$,

$$
\sup _{t \in[0, T]}\left\|R_{2}^{\varepsilon}(t)\right\|_{W \cap L^{2}\left(\mathbb{R}^{d}\right)} \leq C \varepsilon .
$$

To prove this result, we first reduce the analysis to the case of a single oscillation. Decompose $r_{2}^{\varepsilon}$ as

$$
\begin{aligned}
r_{2}^{\varepsilon}= & G\left(\sum_{\ell_{1} \in J} a_{\ell_{1}} e^{i \phi_{\ell_{1}} / \varepsilon}, \ldots, \sum_{\ell_{2 v+1} \in J} a_{\ell_{2 v+1}} e^{i \phi_{\ell_{2 v+1}} / \varepsilon}\right) \\
& -\sum_{j \in J} \sum_{\left(\ell_{1}, \ldots, \ell_{2 v+1}\right) \in I_{j}} G\left(a_{\ell_{1}} e^{i \phi_{\ell_{1}} / \varepsilon}, \ldots, a_{\ell_{2 v+1}} e^{i \phi_{\ell_{2 v+1}} / \varepsilon}\right) \\
= & \sum_{\ell_{1}, \ldots, \ell_{2 v+1} \in J} G\left(a_{\ell_{1}} e^{i \phi_{\ell_{1}} / \varepsilon}, \ldots, a_{\ell_{2 v+1}} e^{i \phi_{\ell_{2 v+1}} / \varepsilon}\right) \\
& -\sum_{j \in J} \sum_{\left(\ell_{1}, \ldots, \ell_{2 v+1} \in I_{j}\right.} G\left(a_{\ell_{1}} e^{i \phi_{\ell_{1}} / \varepsilon}, \ldots, a_{\ell_{2 v+1}} e^{i \phi_{\ell_{2 v+1}} / \varepsilon}\right) \\
= & \sum_{\left(\ell_{1}, \ldots, \ell_{2 v+1}\right) \in N} G\left(a_{\ell_{1}} e^{i \phi_{\ell_{1}} / \varepsilon}, \ldots, a_{\ell_{2 v+1}} e^{i \phi_{\ell_{2 v+1}} / \varepsilon}\right),
\end{aligned}
$$

where

$$
N=J^{2 v+1} \backslash \bigcup_{j \in J} I_{j}
$$

is the nonresonant set. Write

$$
\begin{aligned}
G\left(a_{\ell_{1}} e^{i \ell_{\ell_{1}} / \varepsilon}, \ldots, a_{\ell_{2 v+1}} e^{i \phi_{\ell_{2 v+1}} / \varepsilon}\right) & \\
= & \lambda E\left(a_{\ell_{1}} \bar{a}_{\ell_{2}} \ldots \bar{a}_{\ell_{2 v}} e^{i\left(\phi_{\ell_{1}}-\phi_{\ell_{2}}+\cdots-\phi_{\ell_{2 v}}\right) / \varepsilon}\right) a_{\ell_{2 v+1}} e^{i \phi_{\ell_{2 v+1}} / \varepsilon} \\
& +\mu a_{\ell_{1}} \ldots a_{\ell_{2 v+1}} e^{i\left(\phi_{\ell_{1}}-\phi_{\ell_{2}}+\cdots+\phi_{\ell_{2 v+1}}\right) / \varepsilon},
\end{aligned}
$$


and separate the temporal and spatial oscillations. The nonlocal term reads

$$
\begin{aligned}
\exp \left(-i \sum_{m=1}^{d} \eta_{m}\left(\left|\kappa_{\ell_{1}, m}\right|^{2}-\left|\kappa_{\ell_{2}, m}\right|^{2} \cdots-\left|\kappa_{\ell_{2 v}, m}\right|^{2}\right) t /(2 \varepsilon)\right) \\
\times E\left(a_{\ell_{1}} \bar{a}_{\ell_{2}} \ldots \bar{a}_{\ell_{2 v}} e^{i\left(\kappa_{\ell_{1}}-\kappa_{\ell_{2}}+\cdots-\kappa_{\ell_{2 v}}\right) \cdot x / \varepsilon}\right) a_{\ell_{2 v+1}} e^{i \ell_{\ell_{2 v+1}} \cdot x / \varepsilon},
\end{aligned}
$$

with, since $\left(\ell_{1}, \ldots, \ell_{2 v+1}\right) \in N$,

$$
\sum_{m=1}^{d} \eta_{m}\left(\left|\kappa_{\ell_{1}, m}\right|^{2}-\left|\kappa_{\ell_{2}, m}\right|^{2}+\cdots-\left|\kappa_{\ell_{2 v}, m}\right|^{2}\right) \neq \sum_{m=1}^{d} \eta_{m}\left|\kappa_{\ell_{2 v+1}, m}\right|^{2}
$$

We see that the following lemma is the key:

Lemma 4.6. Let $T>0, \omega \in \mathbb{R}, \kappa_{1}, \kappa_{2} \in \mathbb{R}^{d}$, and $b_{1}, b_{2} \in L^{\infty}\left([0, T] ; W \cap L^{2}\left(\mathbb{R}^{d}\right)\right)$. Denote

$$
D^{\varepsilon}(t, x):=\int_{0}^{t} U^{\varepsilon}(t-\tau)\left(E\left(b_{1}(\tau, x) e^{i \kappa_{1} \cdot x / \varepsilon}\right) b_{2}(\tau, x) e^{i \kappa_{2} \cdot x / \varepsilon} e^{i \omega \tau /(2 \varepsilon)}\right) d \tau .
$$

Let $\kappa=\kappa_{1}+\kappa_{2}$. Assume $\omega \neq|\kappa|^{2}$, and $\partial_{t} b_{j}, \Delta b_{j} \in L^{\infty}\left([0, T] ; W \cap L^{2}\left(\mathbb{R}^{d}\right)\right), j=1,2$. Then

$$
\begin{gathered}
\left\|D^{\varepsilon}\right\|_{X_{T}} \leq \frac{C \varepsilon}{\left.|| \kappa\right|^{2}-\omega \mid}\left(\langle\kappa\rangle^{2}\left\|b_{1} b_{2}\right\|_{X_{T}}+\left\|b_{1} \Delta b_{2}\right\|_{X_{T}}+\left\|b_{2} \Delta b_{1}\right\|_{X_{T}}+\left\|\nabla b_{1} \nabla b_{2}\right\|_{X_{T}}\right. \\
\left.+\left\|b_{1} \partial_{t} b_{2}\right\|_{X_{T}}+\left\|b_{2} \partial_{t} b_{1}\right\|_{X_{T}}\right)
\end{gathered}
$$

where $\|f\|_{X_{T}}:=\|f\|_{L^{\infty}\left([0, T] ; W \cap L^{2}\right)}$, and $C$ is independent of $\kappa_{j}$, $\omega$ and $b_{j}$.

Proof. Let

$$
f^{\varepsilon}(t, x)=E\left(b_{1}(t, x) e^{i \kappa_{1} \cdot x / \varepsilon}\right) b_{2}(t, x) e^{i \kappa_{2} \cdot x / \varepsilon} .
$$

We compute, as in the proof of Lemma 4.3,

$$
\widehat{f}^{\varepsilon}(t, \xi)=(2 \pi)^{-d / 2}\left(\left(\widehat{K}\left(\cdot+\frac{\kappa_{1}}{\varepsilon}\right) \widehat{b}_{1}(t, \cdot)\right) * \widehat{b}_{2}(t, \cdot)\right)\left(\xi-\frac{\kappa}{\varepsilon}\right)=\widehat{g}^{\varepsilon}\left(t, \xi-\frac{\kappa}{\varepsilon}\right),
$$

where

$$
g^{\varepsilon}=E^{\varepsilon}\left(b_{1}\right) b_{2} \quad \text { and } \quad \widehat{E^{\varepsilon}(b)}(\xi)=\widehat{K}\left(\xi+\frac{\kappa_{1}}{\varepsilon}\right) \widehat{b}(\xi) .
$$

By the definition of $U^{\varepsilon}(t)$, we have

$$
\widehat{D}^{\varepsilon}(t, \xi)=\int_{0}^{t} e^{-i \varepsilon(t-\tau)|\xi|^{2} / 2} \widehat{g}^{\varepsilon}\left(t, \xi-\frac{\kappa}{\varepsilon}\right) e^{-i \omega \tau /(2 \varepsilon)} d \tau
$$


Setting $\eta=\xi-\kappa / \varepsilon$, we have

$$
\begin{aligned}
\widehat{D}^{\varepsilon}(t, \xi) & =e^{-i \varepsilon t|\eta+\kappa / \varepsilon|^{2} / 2} \int_{0}^{t} e^{i \varepsilon \tau|\eta+\kappa / \varepsilon|^{2} / 2} \widehat{g}^{\varepsilon}(\tau, \eta) e^{-i \omega \tau /(2 \varepsilon)} d \tau \\
& =e^{-i \varepsilon t|\eta+\kappa / \varepsilon|^{2} / 2} \int_{0}^{t} e^{i \tau \theta / 2} \widehat{g}^{\varepsilon}(\tau, \eta) d \tau
\end{aligned}
$$

where we have denoted

$$
\theta=\varepsilon\left|\eta+\frac{\kappa}{\varepsilon}\right|^{2}-\frac{\omega}{\varepsilon}=\underbrace{\varepsilon|\eta|^{2}+2 \kappa \cdot \eta}_{\theta_{1}}+\underbrace{\frac{|\kappa|^{2}-\omega}{\varepsilon}}_{\theta_{2}} .
$$

Integrate by parts, by first integrating $e^{i \tau \theta_{2} / 2}$ :

$$
\widehat{D}^{\varepsilon}(t, \xi)=-\left.\frac{2 i}{\theta_{2}} e^{i \tau \theta / 2} \widehat{g}^{\varepsilon}(\tau, \eta)\right|_{0} ^{t}+\frac{2 i}{\theta_{2}} \int_{0}^{t} e^{i \tau \theta / 2}\left(i \frac{\theta_{1}}{2} \widehat{g}^{\varepsilon}(\tau, \eta)+\partial_{t} \widehat{g}^{\varepsilon}(\tau, \eta)\right) d \tau .
$$

The lemma follows, since $\widehat{K} \in L^{\infty}\left(\mathbb{R}^{d}\right)$.

In view of Lemma 3.5, Proposition 4.5 follows by summation in Lemma 4.6.

Remark 4.7. Lemma 4.6 remains true if $E$ is replaced by the identity operator. In this case, we simply extend [5, Lemma 5.7] from the $W$ setting to the $W \cap L^{2}$ setting, an extension which requires absolutely no novelty.

\subsection{Proof of Theorem 4.1}

Lemma 3.5 shows that under the assumptions of Theorem 4.1, we also have

$$
\sup _{t \in[0, T]}\left\|r_{3}^{\varepsilon}(t)\right\|_{W \cap L^{2}\left(\mathbb{R}^{d}\right)} \lesssim \varepsilon
$$

Duhamel's formula for the error term $w^{\varepsilon}=u^{\varepsilon}-u_{\text {app }}^{\varepsilon}$ reads

$$
\begin{aligned}
w^{\varepsilon}(t)= & -i \int_{0}^{t} U^{\varepsilon}(t-\tau)\left(G\left(u^{\varepsilon}, \ldots, u^{\varepsilon}\right)-G\left(u_{\mathrm{app}}^{\varepsilon}, \ldots, u_{\mathrm{app}}^{\varepsilon}\right)\right)(\tau) d \tau \\
& -i \int_{0}^{t} U^{\varepsilon}(t-\tau)\left(\lambda r_{1}^{\varepsilon}+r_{2}^{\varepsilon}+r_{3}^{\varepsilon}\right)(\tau) d \tau .
\end{aligned}
$$

We then proceed in two steps:

1. Prove that $w^{\varepsilon}$ is small (as in Theorem 4.1) in $W$.

2. Infer that $w^{\varepsilon}$ is small in $L^{2}\left(\mathbb{R}^{d}\right)$. 
We note the pointwise identity

$$
\begin{aligned}
G\left(u^{\varepsilon}, \ldots, u^{\varepsilon}\right)- & G\left(u_{\mathrm{app}}^{\varepsilon}, \ldots, u_{\mathrm{app}}^{\varepsilon}\right)=\left(\lambda K *\left|u^{\varepsilon}\right|^{2 v}+\mu\left|u^{\varepsilon}\right|^{2 v}\right) w^{\varepsilon} \\
& +\left(\lambda K *\left(\left|u^{\varepsilon}\right|^{2 v}-\left|u_{\mathrm{app}}^{\varepsilon}\right|^{2 v}\right)\right) u_{\mathrm{app}}^{\varepsilon}+\mu\left(\left|u^{\varepsilon}\right|^{2 v}-\left|u_{\mathrm{app}}^{\varepsilon}\right|^{2 v}\right) u_{\mathrm{app}}^{\varepsilon} .
\end{aligned}
$$

Since $\widehat{K} \in L^{\infty}$, we infer

$$
\begin{aligned}
\left\|G\left(u^{\varepsilon}, \ldots, u^{\varepsilon}\right)-G\left(u_{\mathrm{app}}^{\varepsilon}, \ldots, u_{\mathrm{app}}^{\varepsilon}\right)\right\|_{W} \lesssim & \left\|u^{\varepsilon}\right\|_{W}^{2 v}\left\|w^{\varepsilon}\right\|_{W} \\
& +\left(\left\|u^{\varepsilon}\right\|_{W}^{2 \nu-1}+\left\|u_{\mathrm{app}}^{\varepsilon}\right\|_{W}^{2 \nu-1}\right)\left\|w^{\varepsilon}\right\|_{W}\left\|u_{\mathrm{app}}^{\varepsilon}\right\|_{W} \\
\lesssim & \left(\left\|u_{\mathrm{app}}^{\varepsilon}\right\|_{W}^{2 v}+\left\|w^{\varepsilon}\right\|_{W}^{2 v}\right)\left\|w^{\varepsilon}\right\|_{W},
\end{aligned}
$$

where time $t$ is fixed. We know from Lemma 3.4 that $u_{\text {app }}^{\varepsilon} \in C([0, T] ; W)$, so there exists $C_{0}$ independent of $\left.\left.\varepsilon \in\right] 0,1\right]$ such that

$$
\left\|u_{\mathrm{app}}^{\varepsilon}(t)\right\|_{W} \leq C_{0}, \quad \forall t \in[0, T] .
$$

Since $u^{\varepsilon} \in C\left(\left[0, T^{\varepsilon}\right] ; W\right)$ and $w_{\mid t=0}^{\varepsilon}=0$, there exists $t^{\varepsilon}>0$ such that

$$
\left\|w^{\varepsilon}(t)\right\|_{W} \leq C_{0}
$$

for $t \in\left[0, t^{\varepsilon}\right]$. So long as (4.4) holds, we infer

$$
\left\|w^{\varepsilon}(t)\right\|_{W} \lesssim \int_{0}^{t}\left\|w^{\varepsilon}(\tau)\right\|_{W} d \tau+|\lambda| o(1)+\varepsilon,
$$

where we have used Lemma 4.3, Lemma 4.5, and (4.2). The Gronwall lemma implies that so long as (4.4) holds,

$$
\left\|w^{\varepsilon}(t)\right\|_{W} \lesssim|\lambda| o(1)+\varepsilon
$$

where the right hand side does not depend on $t \in[0, T]$. Choosing $\left.\varepsilon \in] 0, \varepsilon_{0}\right]$ with $\varepsilon_{0}$ sufficiently small, we see that (4.4) remains true for $t \in[0, T]$, and the Wiener part of Theorem 4.1 follows.

For the $L^{2}$ setting, we resume (4.3). Plancherel's identity and Young's inequality yield

$$
\begin{aligned}
\left\|G\left(u^{\varepsilon}, \ldots, u^{\varepsilon}\right)-G\left(u_{\mathrm{app}}^{\varepsilon}, \ldots, u_{\mathrm{app}}^{\varepsilon}\right)\right\|_{L^{2}} \lesssim & \left\|u^{\varepsilon}\right\|_{W}^{2 v}\left\|w^{\varepsilon}\right\|_{L^{2}} \\
& +\left(\left\|u^{\varepsilon}\right\|_{W}^{2 \nu-1}+\left\|u_{\mathrm{app}}^{\varepsilon}\right\|_{W}^{2 \nu-1}\right)\left\|w^{\varepsilon}\right\| W\left\|u_{\mathrm{app}}^{\varepsilon}\right\|_{L^{2}} .
\end{aligned}
$$

By Lemma 3.4, $u_{\mathrm{app}}^{\varepsilon} \in C\left([0, T] ; L^{2}\left(\mathbb{R}^{d}\right)\right)$, so by the first part of the proof of Theorem 4.1, the last line in the above inequality is $\lambda o(1)+\mathcal{O}(\varepsilon)$. We also know

$$
\left\|u^{\varepsilon}(t)\right\|_{W} \leq 2 C_{0}, \quad \forall t \in[0, T],
$$

provided $\varepsilon$ is sufficiently small. Gronwall's lemma then yields directly the estimate

$$
\sup _{t \in[0, T]}\left\|w^{\varepsilon}(t)\right\|_{L^{2}} \lesssim|\lambda| o(1)+\varepsilon .
$$

This completes the proof of Theorem 4.1.

Note that for $\lambda=0$, we get the rate $\mathcal{O}(\varepsilon)$ for the remainder term, while for $\lambda \neq 0$, no rate is expected: this follows from the analysis in $\S 4.1$. 


\section{More weakly nonlinear geometric optics}

In this section, we aim to get further insight into the geometric optics approximation in Sobolev spaces of negative order. As we shall see, estimates of the approximate solution (in negative order Sobolev spaces) can be somewhat counter-intuitive. To this end, we consider

$$
i \varepsilon \partial_{t} u^{\varepsilon}+\frac{\varepsilon^{2}}{2} \Delta u^{\varepsilon}=\mu \varepsilon^{J}\left|u^{\varepsilon}\right|^{2 v} u^{\varepsilon}, \quad u^{\varepsilon}(0, x)=\sum_{j \in J_{0}} \alpha_{j}(x) e^{i \kappa_{j} \cdot x / \varepsilon} .
$$

The regime $J=1$ is critical as far as nonlinear effects at leading order are concerned, according to [4]. For $J>1$, nonlinear effects are negligible at leading order in $L^{2} \cap L^{\infty}$. We shall analyze this phenomenon more precisely.

\subsection{Approximate solution}

Pretending that even if $J>1$, the nonlinearity behaves as in the critical case $J=1$, we can resume the discussion from Section 2.1: we consider the same resonant set, and the transport system becomes

$$
\partial_{t} a_{j}^{\varepsilon}+\kappa_{j} \cdot \nabla a_{j}^{\varepsilon}=-i \mu \varepsilon^{J-1} \sum_{\left(\ell_{1}, \ldots, \ell_{2 v+1} \in I_{j}\right.} a_{\ell_{1}}^{\varepsilon} \bar{a}_{\ell_{2}}^{\varepsilon} \ldots a_{\ell_{2 v+1}}^{\varepsilon}, \quad a_{j \mid t=0}^{\varepsilon}=\alpha_{j},
$$

where the notation now emphasizes that the presence of $\varepsilon$ in the equation makes the profiles $\varepsilon$-dependent. Working in the same functional framework as in Section 3, we construct profiles for which we prove first

$$
\left(a_{j}^{\varepsilon}\right)_{j \in J} \in C\left([0, T] ; X\left(\mathbb{R}^{d}\right)\right)
$$

for some $T>0$, uniformly in $\varepsilon \in[0,1]$, then infer

$$
a_{j}^{\varepsilon}(t, x)=\alpha_{j}\left(x-t \kappa_{j}\right)+\mathcal{O}\left(\varepsilon^{J-1}\right) \quad \text { in } C\left([0, T] ; W \cap L^{2}\left(\mathbb{R}^{d}\right)\right) .
$$

On setting

$$
u_{\mathrm{app}}^{\varepsilon}(t, x)=\sum_{j \in J} a_{j}^{\varepsilon}(t, x) e^{i \phi_{j}(t, x) / \varepsilon},
$$

a straightforward adaptation of Theorem 4.1 yields, provided that we start with suitable initial profiles,

$$
\sup _{t \in[0, T]}\left\|u^{\varepsilon}(t)-u_{\mathrm{app}}^{\varepsilon}(t)\right\|_{W \cap L^{2}}=\mathcal{O}(\varepsilon)
$$

\subsection{Negligible or not?}

In view of the proof of the norm inflation phenomenon, we shall now focus on the case of Example 2.3. We know from the above that, starting with three nontrivial $\varepsilon$-oscillations, the zero mode instantaneously appears at order $\varepsilon^{J-1}$. For future reference, we prove a result whose assumptions will become clear later on. 
1912

Rémi Carlos et al.

Lemma 5.1. Let $d \geq 1$ and $\beta>0$. For $f \in \mathcal{S}^{\prime}\left(\mathbb{R}^{d}\right)$ and $\kappa \in \mathbb{R}^{d}$, denote

$$
I^{\varepsilon}(f, \kappa)(x)=f\left(x \varepsilon^{(1-\beta) / 2}\right) e^{i \kappa \cdot x / \varepsilon^{(1+\beta) / 2}} .
$$

(1) Let $\kappa \in \mathbb{R}^{d}$ with $\kappa \neq 0$. For all $\sigma \leq 0$, there exists $C=C(\sigma, \kappa)$ such that for all $f \in \mathcal{S}\left(\mathbb{R}^{d}\right)$,

$$
\left\|I^{\varepsilon}(f, \kappa)\right\|_{H^{\sigma}\left(\mathbb{R}^{d}\right)}^{2} \leq C \varepsilon^{-d(1-\beta) / 2+(1+\beta)|\sigma|}\|f\|_{H^{m}\left(\mathbb{R}^{d}\right)}^{2},
$$

with $m=|\sigma|$ if $\beta \leq 1$, and $m=\frac{1}{2}(1+\beta)|\sigma|$ if $\beta \geq 1$. In addition, $C(\sigma, \kappa) \rightarrow 0$ as $|\kappa| \rightarrow+\infty$.

(2) For all $\sigma \leq 0, \beta<1$ and $f \in L^{2}\left(\mathbb{R}^{d}\right)$,

$$
\left\|I^{\varepsilon}(f, 0)\right\|_{H^{\sigma}\left(\mathbb{R}^{d}\right)}^{2}=\varepsilon^{-d(1-\beta) / 2}\left(\|f\|_{L^{2}\left(\mathbb{R}^{d}\right)}^{2}+o(1)\right) \quad \text { as } \varepsilon \rightarrow 0 .
$$

(3) If $\beta=1, \sigma \in \mathbb{R}$ and $f \in H^{\sigma}\left(\mathbb{R}^{d}\right)$, then $\left\|I^{\varepsilon}(f, 0)\right\|_{H^{\sigma}\left(\mathbb{R}^{d}\right)}^{2}=\|f\|_{H^{\sigma}\left(\mathbb{R}^{d}\right)}^{2}$.

(4) If $\beta>1, \sigma \leq 0$, and $f \in H^{\sigma}\left(\mathbb{R}^{d}\right)$, then

$$
\left\|I^{\varepsilon}(f, 0)\right\|_{H^{\sigma}\left(\mathbb{R}^{d}\right)}^{2} \geq \varepsilon^{-d(1-\beta) / 2+(\beta-1)|\sigma|}\|f\|_{H^{\sigma}\left(\mathbb{R}^{d}\right)}^{2} .
$$

This result shows in particular that for $\sigma \leq 0, \kappa \neq 0$ and $f$ sufficiently smooth, we always have

$$
\left\|I^{\varepsilon}(f, 0)\right\|_{H^{\sigma}\left(\mathbb{R}^{d}\right)} \gg\left\|I^{\varepsilon}(f, \kappa)\right\|_{H^{\sigma}\left(\mathbb{R}^{d}\right)} .
$$

Proof. We compute

$$
\begin{aligned}
\widehat{I^{\varepsilon}(f, \kappa)}(\xi) & =\frac{1}{(2 \pi)^{d / 2}} \int e^{-i x \cdot \xi} f\left(x \varepsilon^{(1-\beta) / 2}\right) e^{i \kappa \cdot x / \varepsilon^{(1+\beta) / 2}} d x \\
& =\varepsilon^{-d(1-\beta) / 2} \frac{1}{(2 \pi)^{d / 2}} \int e^{-i y \cdot \xi / \varepsilon^{(1-\beta) / 2}} f(y) e^{i \kappa \cdot y / \varepsilon} d y \\
& =\varepsilon^{-d(1-\beta) / 2} \widehat{f}\left(\frac{\xi}{\varepsilon^{(1-\beta) / 2}}-\frac{\kappa}{\varepsilon}\right) .
\end{aligned}
$$

Therefore,

$$
\begin{aligned}
\left\|I^{\varepsilon}(f, \kappa)\right\|_{H^{\sigma}\left(\mathbb{R}^{d}\right)}^{2} & =\int\langle\xi\rangle^{2 \sigma}\left|\widehat{I^{\varepsilon}(f, \kappa)}(\xi)\right|^{2} d \xi \\
& =\varepsilon^{-d(1-\beta)} \int\langle\xi\rangle^{2 \sigma}\left|\widehat{f}\left(\frac{\xi}{\varepsilon^{(1-\beta) / 2}}-\frac{\kappa}{\varepsilon}\right)\right|^{2} d \xi .
\end{aligned}
$$

To prove the first point, we write, for $\sigma \leq 0$ and $\beta \leq 1$,

$$
\begin{aligned}
\varepsilon^{d(1-\beta)}\left\|I^{\varepsilon}(f, \kappa)\right\|_{H^{\sigma}\left(\mathbb{R}^{d}\right)}^{2} & \\
& =\int\langle\xi\rangle^{2 \sigma}\left\langle\frac{\xi}{\varepsilon^{(1-\beta) / 2}}-\frac{\kappa}{\varepsilon}\right\rangle^{2 \sigma}\left\langle\frac{\xi}{\varepsilon^{(1-\beta) / 2}}-\frac{\kappa}{\varepsilon}\right)^{2|\sigma|}\left|\widehat{f}\left(\frac{\xi}{\varepsilon^{(1-\beta) / 2}}-\frac{\kappa}{\varepsilon}\right)\right|^{2} d \xi \\
& \leq \sup _{\xi \in \mathbb{R}^{d}}\left(\langle\xi\rangle^{-1}\left\langle\frac{\xi}{\varepsilon^{(1-\beta) / 2}}-\frac{\kappa}{\varepsilon}\right\rangle^{-1}\right)^{2|\sigma|} \varepsilon^{d(1-\beta) / 2}\|f\|_{H^{|\sigma|}\left(\mathbb{R}^{d}\right)}^{2} .
\end{aligned}
$$


Next, write

$$
\left\langle\frac{\xi}{\varepsilon^{(1-\beta) / 2}}-\frac{\kappa}{\varepsilon}\right\rangle^{-1}=\left\langle\varepsilon^{(\beta-1) / 2}\left(\xi-\frac{\kappa}{\varepsilon^{(1+\beta) / 2}}\right)\right\rangle^{-1} \leq\left\langle\xi-\frac{\kappa}{\varepsilon^{(1+\beta) / 2}}\right\rangle^{-1},
$$

where we have used the assumption $\beta \leq 1$. Then use the Peetre inequality (see e.g. [20]) to get the desired estimate in the case $\beta \leq 1$.

In the case $\beta>1$, we use another decomposition:

$$
\begin{aligned}
\varepsilon^{d(1-\beta)} & \left\|I^{\varepsilon}(f, \kappa)\right\|_{H^{\sigma}\left(\mathbb{R}^{d}\right)}^{2} \\
& =\int\langle\xi\rangle^{2 \sigma}\left\langle\frac{\xi}{\varepsilon^{(1-\beta) / 2}}-\frac{\kappa}{\varepsilon}\right\rangle^{(1+\beta) \sigma}\left\langle\frac{\xi}{\varepsilon^{(1-\beta) / 2}}-\frac{\kappa}{\varepsilon}\right)^{(1+\beta)|\sigma|}\left|\widehat{f}\left(\frac{\xi}{\varepsilon^{(1-\beta) / 2}}-\frac{\kappa}{\varepsilon}\right)\right|^{2} d \xi \\
& \leq \sup _{\xi \in \mathbb{R}^{d}}\left(\langle\xi\rangle^{-2}\left\langle\frac{\xi}{\varepsilon^{(1-\beta) / 2}}-\frac{\kappa}{\varepsilon}\right\rangle^{-(1+\beta)}\right)^{|\sigma|} \varepsilon^{d(1-\beta) / 2}\|f\|_{H^{(1+\beta)|\sigma| / 2}\left(\mathbb{R}^{d}\right)}^{2} .
\end{aligned}
$$

We use the obvious estimate

$$
\langle\xi\rangle^{2}\left\langle\frac{\xi}{\varepsilon^{(1-\beta) / 2}}-\frac{\kappa}{\varepsilon}\right\rangle^{1+\beta} \gtrsim \begin{cases}\langle\kappa / \varepsilon\rangle^{1+\beta} & \text { if }|\xi| \leq|\kappa| /\left(2 \varepsilon^{(1+\beta) / 2}\right), \\ \left\langle\kappa / \varepsilon^{(1+\beta) / 2}\right\rangle^{2} & \text { if }|\xi| \geq|\kappa| /\left(2 \varepsilon^{(1+\beta) / 2}\right) .\end{cases}
$$

In both cases, we infer

$$
\langle\xi\rangle^{2}\left\langle\frac{\xi}{\varepsilon^{(1-\beta) / 2}}-\frac{\kappa}{\varepsilon}\right)^{1+\beta} \gtrsim \varepsilon^{-(1+\beta)},
$$

which yields the first point of the lemma. To prove the second point, write

$$
\begin{aligned}
\left\|I^{\varepsilon}(f, 0)\right\|_{H^{\sigma}\left(\mathbb{R}^{d}\right)}^{2} & =\varepsilon^{-d(1-\beta)} \int\langle\xi\rangle^{2 \sigma}\left|\widehat{f}\left(\frac{\xi}{\varepsilon^{(1-\beta) / 2}}\right)\right|^{2} d \xi \\
& =\varepsilon^{-d(1-\beta) / 2} \int\left\langle\varepsilon^{(1-\beta) / 2} \xi\right\rangle^{2 \sigma}|\widehat{f}(\xi)|^{2} d \xi
\end{aligned}
$$

In the case $\beta<1$, we conclude thanks to the Dominated Convergence Theorem. The third point of the lemma $(\beta=1)$ is obvious. To prove the last point, we write,

$$
\begin{aligned}
\left\|I^{\varepsilon}(f, 0)\right\|_{H^{\sigma}\left(\mathbb{R}^{d}\right)}^{2} & =\varepsilon^{-d(1-\beta) / 2} \int\left\langle\varepsilon^{(1-\beta) / 2} \xi\right\rangle^{2 \sigma}|\widehat{f}(\xi)|^{2} d \xi \\
& =\varepsilon^{-d(1-\beta) / 2} \int \frac{1}{\left(1+\varepsilon^{1-\beta}|\xi|^{2}\right)^{|\sigma|}}|\widehat{f}(\xi)|^{2} d \xi \\
& =\varepsilon^{-d(1-\beta) / 2} \int \frac{\varepsilon^{(\beta-1)|\sigma|}}{\left(\varepsilon^{\beta-1}+|\xi|^{2}\right)^{|\sigma|}|\widehat{f}(\xi)|^{2} d \xi} \\
& \geq \varepsilon^{-d(1-\beta) / 2+(\beta-1)|\sigma|} \int \frac{1}{\left(1+|\xi|^{2}\right)^{|\sigma|}}|\widehat{f}(\xi)|^{2} d \xi
\end{aligned}
$$

and the result follows. 
Remark 5.2. The last estimate of Lemma 5.1 is sharp in terms of power of $\varepsilon$, since by dominated convergence

$$
\left\|I^{\varepsilon}(f, 0)\right\|_{H^{\sigma}\left(\mathbb{R}^{d}\right)}^{2} \underset{\varepsilon \rightarrow 0}{\sim} \varepsilon^{-d(1-\beta) / 2+(\beta-1)|\sigma|} \int_{\mathbb{R}^{d}} \frac{1}{|\xi|^{2|\sigma|}}|\widehat{f}(\xi)|^{2} d \xi
$$

for all $f \in L^{2} \cap H^{\sigma}$ when $-d / 2<\sigma<0$, and for all $f \in L^{2} \cap H^{\sigma}$ such that $0 \notin \operatorname{supp} \widehat{f}$ when $\sigma \leq-d / 2$.

Next, we shall simply apply Lemma 5.1 (in the case $\beta=1$ ) to $u_{\mathrm{app}}^{\varepsilon}$. We find, thanks to Lemma 2.4,

$$
\left\|a_{0}^{\varepsilon}(t)\right\|_{H^{s}\left(\mathbb{R}^{d}\right)} \approx \varepsilon^{J-1}
$$

for $t>0$ arbitrarily small. On the other hand, the first point of Lemma 5.1 yields, for $s \leq 0$,

We infer, if $s \leq 0$,

$$
\left\|u_{\mathrm{app}}^{\varepsilon}(t)-a_{0}^{\varepsilon}(t)\right\|_{H^{s}\left(\mathbb{R}^{d}\right)} \lesssim \varepsilon^{|s|} .
$$

$$
\left\|u^{\varepsilon}(t)\right\|_{H^{s}\left(\mathbb{R}^{d}\right)}=\left\|a_{0}^{\varepsilon}(t)\right\|_{H^{s}\left(\mathbb{R}^{d}\right)}+\mathcal{O}\left(\varepsilon^{|s|}\right)+\mathcal{O}(\varepsilon),
$$

where the last term stems from the geometric optics approximation, and the simple control, for $s \leq 0,\|f\|_{H^{s}} \leq\|f\|_{L^{2}}$. We conclude that for $t>0$ arbitrarily small, the zero mode is not negligible in $H^{s}\left(\mathbb{R}^{d}\right)$, provided

$$
|s|>J-1 \text { and } J-1<1, \quad \text { that is, } \quad s<1-J<0 \text { and } J<2 .
$$

We finally remark that having the zero mode not negligible at leading order means that nonlinear effects are present at leading order, in $H^{s}\left(\mathbb{R}^{d}\right)$. We summarize these remarks in the following

Proposition 5.3. Let $s, J \in \mathbb{R}$ satisfy $s<1-J<0$ and $J<2$. Set $J_{0}=\{1,2,3\}$, $\kappa_{0}=0_{\mathbb{R}^{d}}$, and consider $\Phi_{0}$ from (2.7). Then there exist $\alpha_{1}, \alpha_{2}, \alpha_{3} \in \mathcal{S}\left(\mathbb{R}^{d}\right)$, independent of $s$ and $J$, and $a T>0$, such that the unique solution $u^{\varepsilon} \in C\left([0, T] ; L^{2} \cap L^{\infty}\right)$ to (5.1) satisfies, for all $t \in] 0, T]$ where $a_{0}^{\varepsilon}(t) \neq 0$,

$$
\left\|u^{\varepsilon}(t)\right\|_{H^{s}\left(\mathbb{R}^{d}\right)} \approx\left\|a_{0}^{\varepsilon}(t)\right\|_{H^{s}\left(\mathbb{R}^{d}\right)} \approx \varepsilon^{J-1} \gg\left\|u^{\varepsilon}(0)\right\|_{H^{s}\left(\mathbb{R}^{d}\right)} \approx \varepsilon^{|s|} \quad \text { as } \varepsilon \rightarrow 0 .
$$

\section{Norm inflation}

To explain our approach, we first consider the nonlinear Schrödinger equation

$$
i \partial_{t} \psi+\frac{1}{2} \Delta \psi=\mu|\psi|^{2 v} \psi, \quad x \in \mathbb{R}^{d}, \quad \psi_{\mid t=0}=\varphi .
$$

We proceed in four steps:

1. Choice of a suitable scaling in order to be able to use weakly nonlinear geometric optics.

2. Link between the Sobolev norms of $\psi$ and approximate solutions given by geometric optics.

3. High frequency analysis (WNLGO).

4. Conclusion: what WNLGO implies in terms of $\psi$. 


\subsection{Scaling}

We consider the general scaling

$$
u^{\varepsilon}(t, x)=\varepsilon^{\alpha} \psi\left(\varepsilon^{\beta} t, \varepsilon^{\gamma} x\right)
$$

To simplify the discussion, we want to fix $\alpha, \beta, \gamma$ so that $\psi$ solves (6.1) and $u^{\varepsilon}$ solves

$$
i \varepsilon \partial_{t} u^{\varepsilon}+\frac{\varepsilon^{2}}{2} \Delta u^{\varepsilon}=\mu \varepsilon^{J}\left|u^{\varepsilon}\right|^{2 v} u^{\varepsilon}
$$

with $1 \leq J<2$. We will relate phenomena affecting $u^{\varepsilon}$ for times of order $\mathcal{O}(1)$ with a norm inflation for $\psi$ on times of order $o(1)$ : this imposes $\beta>0$. We find the relation

$$
1+\beta=2+2 \gamma=J+2 v \alpha .
$$

Leaving only $\beta$ as a free parameter, this means

$$
u^{\varepsilon}(t, x)=\varepsilon^{(\beta+1-J) /(2 v)} \psi\left(\varepsilon^{\beta} t, \varepsilon^{(\beta-1) / 2} x\right) .
$$

The initial data that we want to consider for $u^{\varepsilon}$ are

$$
u^{\varepsilon}(0, x)=\sum_{j \in J_{0}} \alpha_{j}(x) e^{i \kappa_{j} \cdot x / \varepsilon}
$$

with $\kappa_{j} \in \mathbb{R}^{d}$ and $\alpha_{j} \in \mathcal{S}\left(\mathbb{R}^{d}\right)$. In view of (6.2), this yields

$$
\psi(0, x)=\varepsilon^{-(\beta+1-J) /(2 v)} \sum_{j \in J_{0}} \alpha_{j}\left(x \varepsilon^{(1-\beta) / 2}\right) e^{i \kappa_{j} \cdot x / \varepsilon^{(1+\beta) / 2}} .
$$

This is exactly the scaling used in Lemma 5.1, up to the factor $\varepsilon^{-(\beta+1-J) /(2 v)}$.

\subsection{High frequency analysis}

We resume the framework of Example 2.3, and suppose that at time $t=0, u^{\varepsilon}$ is the sum of three plane waves:

$$
u^{\varepsilon}(0, x)=\sum_{j=1}^{3} \alpha_{j}(x) e^{i \kappa_{j} \cdot x / \varepsilon}
$$

with $\alpha_{1}, \alpha_{2}, \alpha_{3} \in \mathcal{S}\left(\mathbb{R}^{d}\right)$ and

$$
\kappa_{1}=(1,0, \ldots, 0), \kappa_{2}=(1,1,0, \ldots, 0), \kappa_{3}=(0,1,0, \ldots, 0) \in \mathbb{R}^{d} .
$$

The important point is that by nonlinear resonance, the zero mode appears (and possibly other modes):

$$
u_{\mathrm{app}}^{\varepsilon}(t, x)=a_{0}(t, x)+\sum_{j=1}^{\infty} a_{j}(t, x) e^{i \phi_{j}(t, x) / \varepsilon}, \quad \phi_{j}(t, x)=\kappa_{j} \cdot x-\frac{t}{2}\left|\kappa_{j}\right|^{2},
$$


where, for $j \geq 1$, we have $\kappa_{j} \in \mathbb{Z}^{d} \backslash\{0\}$, and the series is convergent in $L^{2}\left(\mathbb{R}^{d}\right)$, and more generally in all Sobolev spaces from Remark 3.6. By Lemma 2.4 (or Lemma 2.11, or Lemma 2.13), even though $a_{0}$ is zero at time $t=0$, we can choose initial profiles so that $\partial_{t} a_{0 \mid t=0} \neq 0$ : this mode becomes instantaneously nontrivial. Geometric optics yields

$$
\left\|u^{\varepsilon}-u_{\mathrm{app}}^{\varepsilon}\right\|_{L^{\infty}\left([0, T] ; L^{2}\left(\mathbb{R}^{d}\right)\right)} \underset{\varepsilon \rightarrow 0}{\longrightarrow} 0 .
$$

Below, we take advantage of this approximation, and of the fact that the new (nonoscillating) generated mode $a_{0}$ is much larger than the others in negative order Sobolev spaces, as measured by Lemma 5.1.

\subsection{Proof of Theorem 1.11}

In this case, we choose $J=1$. The reason why we have no flexibility for $J$ here is that in Section 5, we have used the fact that a rate for the error estimate is available, $\left\|u^{\varepsilon}-u_{\text {app }}^{\varepsilon}\right\|_{L^{\infty}\left([0, T], W \cap L^{2}\right)}=\mathcal{O}(\varepsilon)$. Unlike the (NLS) case, no rate is available in general in the presence of a nonlocal term; see Section 4.1.

For $\varepsilon=1 / n$, denote by $\psi_{n}$ the solution given by (6.2), and by $\varphi_{n}$ its trace at $t=0$. Lemma 5.1 yields, for $s<0$,

$$
\left\|\varphi_{n}\right\|_{H^{s}\left(\mathbb{R}^{d}\right)}^{2} \lesssim \varepsilon^{-\beta / \nu-d(1-\beta) / 2+|s|(1+\beta)} .
$$

We have $\left\|\varphi_{n}\right\|_{H^{s}} \rightarrow 0$ provided

$$
-\frac{\beta}{v}-d \frac{1-\beta}{2}+|s|(1+\beta)>0 \Leftrightarrow \beta>\frac{d / 2-|s|}{s_{c}+|s|},
$$

where $s_{c}$, given by (1.1), is always nonnegative in the framework of this paper.

Let $\tau>0$ independent of $\varepsilon$ be such that $a_{0}(\tau) \neq 0$. Set $t_{n}=\tau \varepsilon^{\beta}=\tau / n^{\beta}$; then $t_{n} \rightarrow 0$ provided $\beta>0$. Denote by $\psi_{\text {app }}$ the function obtained from $u_{\text {app }}^{\varepsilon}$ via the scaling (6.2) (the dependence upon $n$ is omitted to ease the notation). Consider $\sigma \leq 0$. We have obviously

$$
\left\|\psi_{n}\left(t_{n}\right)-\psi_{\mathrm{app}}\left(t_{n}\right)\right\|_{H^{\sigma}\left(\mathbb{R}^{d}\right)} \leq\left\|\psi_{n}\left(t_{n}\right)-\psi_{\mathrm{app}}\left(t_{n}\right)\right\|_{L^{2}\left(\mathbb{R}^{d}\right)} .
$$

Estimate (6.3) shows that

$$
\left\|\psi_{n}\left(t_{n}\right)-\psi_{\mathrm{app}}\left(t_{n}\right)\right\|_{L^{2}\left(\mathbb{R}^{d}\right)}=o\left(\left\|\psi_{\mathrm{app}}\left(t_{n}\right)\right\|_{L^{2}\left(\mathbb{R}^{d}\right)}\right) \quad \text { as } n \rightarrow \infty .
$$

We assume $0<\beta \leq 1$. Lemma 5.1 yields, for $\beta<1$,

$$
\left\|\psi_{\text {app }}\left(t_{n}\right)\right\|_{H^{\sigma}\left(\mathbb{R}^{d}\right)}^{2} \underset{\varepsilon \rightarrow 0}{\sim}\left\|\psi_{\text {app }}\left(t_{n}\right)\right\|_{L^{2}\left(\mathbb{R}^{d}\right)}^{2} \underset{\varepsilon \rightarrow 0}{\sim} \varepsilon^{-\beta / \nu-d(1-\beta) / 2}\left\|a_{0}(\tau)\right\|_{L^{2}\left(\mathbb{R}^{d}\right)}^{2} .
$$

For $\beta=1$, we still have

$$
\left\|\psi_{\mathrm{app}}\left(t_{n}\right)\right\|_{H^{\sigma}\left(\mathbb{R}^{d}\right)}^{2} \approx \varepsilon^{-\beta / \nu-d(1-\beta) / 2} \approx\left\|\psi_{\mathrm{app}}\left(t_{n}\right)\right\|_{L^{2}\left(\mathbb{R}^{d}\right)}^{2} .
$$

We infer, for $\beta \leq 1$,

$$
\left\|\psi_{n}\left(t_{n}\right)\right\|_{H^{\sigma}\left(\mathbb{R}^{d}\right)}^{2} \underset{\varepsilon \rightarrow 0}{\sim}\left\|\psi_{\mathrm{app}}\left(t_{n}\right)\right\|_{H^{\sigma}\left(\mathbb{R}^{d}\right)}^{2} \approx \varepsilon^{-\beta / \nu-d(1-\beta) / 2} .
$$


This power of $\varepsilon$ is always negative, since we have $s_{c} \geq 0$, and $\beta s_{c} \leq s_{c}<d / 2$. So to prove norm inflation, we simply have to check the compatibility of (6.4) with the condition $0<\beta \leq 1$ :

$$
\frac{d / 2-|s|}{s_{c}+|s|}<1 \Leftrightarrow|s|>\frac{1}{2 v} .
$$

The case of equality, which corresponds to the statement of Theorem 1.11, can be reached thanks to logarithmic modifications (multiply the initial data by $\ln \varepsilon$ ), in the same spirit as in $[7,2]$.

Finally, we simply note that all the negative order Sobolev norms of $\psi$ become unbounded along the sequence of times $t_{n}$. It is then obvious that so do the positive order Sobolev norms.

\subsection{Proof of Theorem 1.13}

We now assume $\lambda=0$. There is no nonlocal term and we can use the analysis of Section 5 with $1 \leq J<2$. We mimic the discussion from the previous subsection concerning the algebraic requirements on the different parameters, $\beta, s$, and now $J$. Lemma 5.1 yields, for $s \leq 0$,

$$
\left\|\varphi_{n}\right\|_{H^{s}\left(\mathbb{R}^{d}\right)}^{2} \lesssim \varepsilon^{-(\beta+1-J) / \nu-d(1-\beta) / 2+|s|(1+\beta)},
$$

so we demand

$$
\beta>\frac{d / 2-|s|-(J-1) / v}{s_{c}+|s|} .
$$

We will still demand $0<\beta \leq 1$, so for all $\sigma \in \mathbb{R}$,

$$
\left\|\psi_{n}\left(t_{n}\right)\right\|_{H^{\sigma}\left(\mathbb{R}^{d}\right)}^{2} \underset{\varepsilon \rightarrow 0}{\sim}\left\|\psi_{\mathrm{app}}\left(t_{n}\right)\right\|_{H^{\sigma}\left(\mathbb{R}^{d}\right)}^{2} \approx \varepsilon^{2(J-1)} \varepsilon^{-(\beta+1-J) / \nu-d(1-\beta) / 2},
$$

where the new term $\varepsilon^{2(J-1)}$ is due to the fact that we consider "more weakly" nonlinear geometric optics. This total power of $\varepsilon$ is negative provided

$$
\beta s_{c}<\frac{d}{2}-(J-1)\left(2+\frac{1}{v}\right) .
$$

The algebraic requirements are $0<\beta \leq 1,1 \leq J<2$, (6.5), and (6.6). We check that they are compatible, provided $s<-1 /(1+2 v)$. For such an $s$, we can find $\delta>0$ so that

$$
s=-\frac{\delta}{v}-\frac{1}{1+2 v} \text {. }
$$

$\operatorname{Pick} \beta=1$ and

$$
J=\frac{2+2 v}{1+2 v}-\delta
$$

The first two conditions are obviously fulfilled, at least if $0<\delta \ll 1$ (it suffices to prove Theorem 1.13 for $s$ close to $-1 /(1+2 v))$. A direct computation shows that so are $(6.5)$ and (6.6). Theorem 1.13 follows. 


\section{Appendix A. Proof of Proposition 1.9}

Without recalling all details of [3, Proposition 1], we shall give a flavor of this rather general result, and explain how to infer Proposition 1.9. Roughly speaking, it suffices to prove that one term in the Picard iteration process rules out the condition in Definition 1.1, in order to deny well-posedness for the solution to the nonlinear problem. Therefore, we start with the free equation

$$
i \partial_{t} \psi+\frac{1}{2} \Delta_{\eta} \psi=0, \quad \psi_{\mid t=0}=\varphi .
$$

For (NLS), we then consider the integral term

$$
D(\varphi)(t, x)=-i \mu \int_{0}^{t} e^{i \frac{t-\tau}{2} \Delta_{\eta}}\left(|\psi|^{2 v} \psi\right)(\tau, x) d \tau .
$$

To prove Proposition 1.9, it suffices to show that the map $\varphi \mapsto D(\varphi)$ is not continuous from $H^{s}\left(\mathbb{R}^{d}\right)$ to $C\left([0, T] ; H^{\sigma}\left(\mathbb{R}^{d}\right)\right)$, that is, there is no control such as

$$
\|D(\varphi)\|_{L^{\infty}\left([0, T] ; H^{\sigma}\left(\mathbb{R}^{d}\right)\right)} \lesssim\|\varphi\|_{H^{s}\left(\mathbb{R}^{d}\right)}^{2 v+1} .
$$

The main difference with the approach of Section 6 is that now the analysis is "much more linear". In practice, we resume the same lines as in Section 6, up to the factor $\varepsilon^{(\beta+1-J) /(2 v)}$, which was there only to get precisely a weakly nonlinear regime. We also fix $\beta=1$, and consider

$$
\varphi(x)=\sum_{j=1}^{3} \alpha_{j}(x) e^{i \kappa_{j} \cdot x / \varepsilon},
$$

where $\alpha_{j} \in \mathcal{S}\left(\mathbb{R}^{d}\right)$ and the $\kappa_{j}$ 's are given by Example 2.3. By creation of the zero mode (from Lemma 2.4), and Lemma 5.1, (A.1) would imply

$$
1 \lesssim \varepsilon^{-s(2 v+1)}
$$

which is impossible if $s<0$.

In the case where the nonlocal term is present, one can argue along the same lines. For (DS), the assumption $\lambda+2 \mu \neq 0$ arises when one wants to use Lemma 2.11 in place of Lemma 2.4. For (DGP), one uses Lemma 2.14.

\section{Appendix B. On negative order Sobolev spaces}

Lemma 5.1 with $\beta=1$ shows that all Sobolev norms for $I^{\varepsilon}(f, \kappa)=f(x) e^{i \kappa \cdot x / \varepsilon}$ behave according to the intuition as $\varepsilon \rightarrow 0$, provided $f \in \mathcal{S}\left(\mathbb{R}^{d}\right)$, that is,

$$
\left\|I^{\varepsilon}(f, \kappa)\right\|_{H^{s}\left(\mathbb{R}^{d}\right)} \lesssim \varepsilon^{-s}, \quad \forall s \in \mathbb{R}, \text { if } \kappa \neq 0 .
$$

The aim of this appendix is to show that in general, negative order Sobolev norms can behave rather strangely on functions which exhibit rapid oscillations and/or concentration effects (as is typically the case for wave functions of quantum mechanics in the semiclassical limit). 
Example B.1 (Oscillatory functions). We consider a WKB state with nonlinear phase function $\phi(x)=-\frac{1}{2}|x|^{2}$ :

$$
g^{\varepsilon}(x)=e^{-|x|^{2} / 2} e^{-i|x|^{2} /(2 \varepsilon)}, \quad x \in \mathbb{R}^{d} .
$$

Lemma B.2. Let $d \geq 1$. Then as $\varepsilon \rightarrow 0$,

$$
\left\|g^{\varepsilon}\right\|_{H^{s}\left(\mathbb{R}^{d}\right)} \approx \begin{cases}\varepsilon^{-s} & \text { if } s>-d / 2, \\ \varepsilon^{d / 2} \sqrt{\log (1 / \varepsilon)} & \text { if } s=-d / 2, \\ \varepsilon^{d / 2} & \text { if } s<-d / 2 .\end{cases}
$$

Remark B.3. The same asymptotic result is true in homogeneous Sobolev spaces (it will be clear from the proof), but the result in the inhomogeneous case is perhaps more striking.

Proof. Consider more generally, for $z \in \mathbb{C}$ with $\operatorname{Re} z>0$,

$$
g_{z}(x)=e^{-z|x|^{2} / 2} .
$$

We compute

$$
\mathcal{F} g_{z}(\xi)=z^{-d / 2} e^{-|\xi|^{2} /(2 z)}
$$

For $s \in \mathbb{R}$, we have, if $z=a+i b, a, b \in \mathbb{R}, a>0$,

$$
\begin{aligned}
\left\|g_{z}\right\|_{H^{s}\left(\mathbb{R}^{d}\right)}^{2} & =\int_{\mathbb{R}^{d}}\langle\xi\rangle^{2 s}\left|\mathcal{F} g_{z}(\xi)\right|^{2} d \xi=\frac{1}{|z|^{d}} \int_{\mathbb{R}^{d}}\langle\xi\rangle^{2 s} e^{-\frac{a}{a^{2}+b^{2}}|\xi|^{2}} d \xi \\
& =\frac{1}{a^{d / 2}} \int_{\mathbb{R}^{d}}\left\langle\left(\frac{a^{2}+b^{2}}{a}\right)^{1 / 2} \eta\right\rangle^{2 s} e^{-|\eta|^{2}} d \eta .
\end{aligned}
$$

In the present case of $z=1+i / \varepsilon$,

$$
\begin{aligned}
\left\|g^{\varepsilon}\right\|_{H^{s}\left(\mathbb{R}^{d}\right)}^{2} & =\int_{\mathbb{R}^{d}}\left\langle\left(1+\frac{1}{\varepsilon^{2}}\right)^{1 / 2} \eta\right\rangle^{2 s} e^{-|\eta|^{2}} d \eta \\
& \approx \int_{\mathbb{R}^{d}}\left\langle\frac{\eta}{\varepsilon}\right\rangle^{2 s} e^{-|\eta|^{2}} d \eta=c(d) \int_{0}^{+\infty}\left(1+\frac{r^{2}}{\varepsilon^{2}}\right)^{s} e^{-r^{2}} r^{d-1} d r .
\end{aligned}
$$

We split the last integral into $\int_{0}^{\varepsilon}+\int_{\varepsilon}^{+\infty}$. Then we have for all $s$, since $1 \leq 1+r^{2} / \varepsilon^{2} \leq 2$ in the first integral,

$$
\int_{0}^{\varepsilon}\left(1+\frac{r^{2}}{\varepsilon^{2}}\right)^{s} e^{-r^{2}} r^{d-1} d r \approx \int_{0}^{\varepsilon} e^{-r^{2}} r^{d-1} d r \approx \varepsilon^{d}
$$

For the other term, since $r^{2} / \varepsilon^{2} \leq 1+r^{2} / \varepsilon^{2} \leq 2 r^{2} / \varepsilon^{2}$, we have

$$
I(\varepsilon):=\int_{\varepsilon}^{+\infty}\left(1+\frac{r^{2}}{\varepsilon^{2}}\right)^{s} e^{-r^{2}} r^{d-1} d r \approx \varepsilon^{-2 s} \int_{\varepsilon}^{+\infty} e^{-r^{2}} r^{2 s+d-1} d r .
$$


Therefore, if $d+2 s>0$, then $I(\varepsilon) \approx \varepsilon^{-2 s}$. On the other hand, if $d+2 s<0$, the lack of integrability near the origin yields

$$
I(\varepsilon) \approx \varepsilon^{d}
$$

and if $d+2 s=0$, we have, for the same reason,

$$
I(\varepsilon) \approx \varepsilon^{-2 s} \log \frac{1}{\varepsilon}=\varepsilon^{d} \log \frac{1}{\varepsilon} .
$$

The lemma follows.

Example B.4 (Concentrating functions). Another important example concerns functions which concentrate at a point, e.g.

$$
p^{\varepsilon}(x)=\varepsilon^{-d / 4} e^{-|x|^{2} /(2 \varepsilon)}, \quad x \in \mathbb{R}^{d} .
$$

The function $p^{\varepsilon}$ is a so-called coherent state in quantum mechanics (centered at the origin in the phase space).

Lemma B.5. Let $d \geq 1$. Then

$$
\left\|p^{\varepsilon}\right\|_{H^{s}\left(\mathbb{R}^{d}\right)} \approx \begin{cases}\varepsilon^{-s / 2} & \text { if } s>-d / 2, \\ \varepsilon^{d / 4} \sqrt{\log (1 / \varepsilon)} & \text { if } s=-d / 2, \\ \varepsilon^{d / 4} & \text { if } s<-d / 2 .\end{cases}
$$

Proof. Resume the above computation, with now $a=1 / \varepsilon$ and $b=0$. We have

$$
\left\|p^{\varepsilon}\right\|_{H^{s}\left(\mathbb{R}^{d}\right)}^{2}=\int_{\mathbb{R}^{d}}\left\langle\frac{\eta}{\sqrt{\varepsilon}}\right\rangle^{2 s} e^{-|\eta|^{2}} d \eta .
$$

We can then resume the same computations, by simply replacing $\varepsilon$ with $\sqrt{\varepsilon}$.

Acknowledgments. This work was supported by the French ANR project R.A.S. (ANR-08-JCJC0124-01) and by the Royal Society Research fellowship of C. Sparber. The authors wish to thank the referee for the thorough analysis of the text.

\section{References}

[1] Ablowitz, M. J., Clarkson, P. A.: Solitons, Nonlinear Evolution Equations and Inverse Scattering. London Math. Soc. Lecture Note Ser. 149, Cambridge Univ. Press (1991) Zbl 0762.35001 MR 1149378

[2] Alazard, T., Carles, R.: Loss of regularity for super-critical nonlinear Schrödinger equations. Math. Ann. 343, 397-420 (2009) Zbl 1161.35047 MR 2461259

[3] Bejenaru, I., Tao, T.: Sharp well-posedness and ill-posedness results for a quadratic nonlinear Schrödinger equation. J. Funct. Anal. 233, 228-259 (2005) Zbl 1090.35162 MR 2204680

[4] Carles, R., Semi-Classical Analysis for Nonlinear Schrödinger Equations. World Sci., Hackensack, NJ (2008) Zbl 1153.35070 MR 2406566 
[5] Carles, R., Dumas, E., Sparber, C.: Multiphase weakly nonlinear geometric optics for Schrödinger equations. SIAM J. Math. Anal. 42, 489-518 (2010) Zbl 1153.35070 MR 2607351

[6] Carles, R., Markowich, P. A., Sparber, C.: On the Gross-Pitaevskii equation for trapped dipolar quantum gases. Nonlinearity 21, 2569-2590 (2008) Zbl 1157.35102 MR 2448232

[7] Christ, M., Colliander, J., Tao, T.: Ill-posedness for nonlinear Schrödinger and wave equations. arXiv:math/0311048

[8] Christ, M., Colliander, J., Tao, T.: Instability of the periodic nonlinear Schrödinger equation. arXiv:math/0311227

[9] Colin, M., Lannes, D.: Short pulses approximations in dispersive media. SIAM J. Math. Anal. 41, 708-732 (2009) Zbl 1200.35182 MR 2515782

[10] Colliander, J., Keel, M., Staffilani, G., Takaoka, H., Tao, T.: Transfer of energy to high frequencies in the cubic defocusing nonlinear Schrödinger equation. Invent. Math. 181, 39-113 (2010) Zbl 1197.35265 MR 2651381

[11] Gan, Z., Zhang, J.: Sharp threshold of global existence and instability of standing wave for a Davey-Stewartson system. Comm. Math. Phys. 283, 93-125 (2008) Zbl 1158.35416 MR 2430630

[12] Ghidaglia, J.-M., Saut, J.-C.: On the initial value problem for the Davey-Stewartson systems. Nonlinearity 3, 475-506 (1990) Zbl 0727.35111 MR 1054584

[13] Giannoulis, J., Mielke, A., Sparber, C.: High-frequency averaging in semi-classical Hartreetype equations. Asymptot. Anal. 70, 87-100 (2010) Zbl 1229.35261 MR 2731651

[14] Kenig, C., Ponce, G., Vega, L.: On the ill-posedness of some canonical dispersive equations. Duke Math. J. 106, 617-633 (2001) Zbl 1034.35145 MR 1813239

[15] Lebeau, G.: Perte de régularité pour les équations d'ondes sur-critiques. Bull. Soc. Math. France 133, 145-157 (2005) Zbl 1071.35020 MR 2145023

[16] Molinet, L.: On ill-posedness for the one-dimensional periodic cubic Schrödinger equation. Math. Res. Lett. 16, 111-120 (2009) Zbl 1180.35487 MR 2480565

[17] Panthee, M.: On the ill-posedness result for the BBM equation. Discrete Contin. Dynam. Systems 30, 253-259 (2011) Zbl 1214.35060 MR 2773142

[18] Sulem, C., Sulem, P.-L.: The Nonlinear Schrödinger Equation, Self-Focusing and Wave Collapse. Springer, New York (1999) Zbl 0928.35157 MR 1696311

[19] Thomann, L.: Instabilities for supercritical Schrödinger equations in analytic manifolds. J. Differential Equations 245, 249-280 (2008) Zbl 1157.35107 MR 2422717

[20] Trèves, F.: Introduction to Pseudodifferential and Fourier Integral Operators. Vol. 1. Plenum Press, New York (1980) Zbl 0453.47027 MR 0597144

[21] Yi, S., You, L.: Trapped atomic condensates with anisotropic interactions. Phys. Rev. A 61, no. 4,041604 (2000) 